\title{
Assessment and adjustment of approximate inference algorithms using the law of total variance
}

\author{
Xuejun Yu. .* David J. Nott. $\|$ Minh-Ngoc Tran and Nadja Klein
}

\begin{abstract}
A common method for assessing validity of Bayesian sampling or approximate inference methods makes use of simulated data replicates for parameters drawn from the prior. Under continuity assumptions, quantiles of functions of the simulated parameter values within corresponding posterior distributions are uniformly distributed. Checking for uniformity when a posterior density is approximated numerically provides a diagnostic for algorithm validity. Furthermore, adjustments to achieve uniformity can improve the quality of approximate inference methods. A weakness of this general approach is that it seems difficult to extend beyond scalar functions of interest. The present article develops an alternative to quantile-based checking and adjustment methods which is inherently multivariate. The new approach is based on use of the tower property of conditional expectation and the law of total variance for relating prior and posterior expectations and covariances. For adjustment, approximate inferences are modified so that the correct prior to posterior relationships hold. We illustrate the method in three examples. The first uses an auxiliary model in a likelihood-free inference problem. The second considers corrections for variational Bayes approximations in a deep neural network generalized linear mixed model. Our final application considers a deep neural network surrogate for approximating Gaussian process regression predictive inference.
\end{abstract}

Keywords: Law of total variance, Likelihood-free inference, Markov chain Monte Carlo, Variational approximation.

\footnotetext{
* Department of Statistics and Applied Probability, National University of Singapore

†Corresponding author: standj@nus.edu.sg. Department of Statistics and Applied Probability, National University of Singapore and Institute of Operations Research and Analytics, National University of Singapore. ${ }^{\ddagger}$ Discipline of Business Analytics, The University of Sydney Business School, Australian Centre of Excellence for Mathematical and Statistical Frontiers (ACEMS)

${ }^{\S}$ School of Business and Economics, Statistics, Humboldt-Universität zu Berlin.
} 


\section{Introduction}

Researchers are increasingly called upon to analyse large datasets using complex models, and this has resulted in approximate inference methods being more widely used, such as likelihoodfree inference (Marin et al., 2012; Blum et al., 2013) and variational inference (Ormerod and Wand, 2010; Blei et al., 2017). However, often the accuracy of these methods cannot be guaranteed, so there is interest in diagnosing algorithm failures, and in developing adjustments which improve their performance. The current paper is a contribution to this area, and considers an approach that can easily handle multivariate quantities of interest, unlike the existing approaches which are hard to extend beyond checking and adjustment for univariate quantities.

A common method for checking Bayesian computational algorithms is based on posterior quantiles (Cook et al., 2006; Gelman, 2017). To save notation, and following the discussion in Cook et al. (2006), consider the case of a scalar parameter $\theta$ of interest. The data will be denoted as $\boldsymbol{y}$. Suppose we have a joint Bayesian model for $(\theta, \boldsymbol{y})$ specified by a density $p(\theta, \boldsymbol{y})=p(\theta) p(\boldsymbol{y} \mid \theta)$, where $p(\theta)$ is the prior density and $p(\boldsymbol{y} \mid \theta)$ is the sampling density. Consider a simulation $\left(\theta^{\prime}, \boldsymbol{y}^{\prime}\right)$ from $p(\theta, \boldsymbol{y})$, obtained by drawing $\theta^{\prime} \sim p(\theta)$, and then $\boldsymbol{y}^{\prime} \sim$ $p\left(\boldsymbol{y} \mid \theta^{\prime}\right)$. Noting that $p(\theta) p(\boldsymbol{y} \mid \theta)=p(\boldsymbol{y}) p(\theta \mid \boldsymbol{y})$, we can equivalently think of $\left(\theta^{\prime}, \boldsymbol{y}^{\prime}\right)$ as obtained by sampling $\boldsymbol{y}^{\prime} \sim p(\boldsymbol{y})$, then $\theta^{\prime} \sim p\left(\theta \mid \boldsymbol{y}^{\prime}\right)$, so that we can regard $\theta^{\prime}$ in the joint sample as being an exact draw from $p\left(\theta \mid \boldsymbol{y}^{\prime}\right)$. Under continuity assumptions, transforming a random variable by its distribution function gives a uniform random variable, so writing $F\left(\theta \mid \boldsymbol{y}^{\prime}\right)$ for the posterior distribution function of $\theta$ given $\boldsymbol{y}^{\prime}$, we have that $F\left(\theta^{\prime} \mid \boldsymbol{y}^{\prime}\right) \sim U[0,1]$, where $U[0,1]$ is the uniform density on $[0,1]$. When Markov chain Monte Carlo (MCMC) simulation or some approximate inference algorithm is used to approximate $F\left(\theta \mid \boldsymbol{y}^{\prime}\right)$ by $\tilde{F}\left(\theta \mid \boldsymbol{y}^{\prime}\right)$ say, testing for uniformity of $\tilde{F}\left(\theta^{\prime} \mid \boldsymbol{y}^{\prime}\right)$ based on replicate samples of $\left(\theta^{\prime}, \boldsymbol{y}^{\prime}\right)$ can be used to diagnose problems with the simulation algorithm, or to make inference adjustments.

Gelman (2017) points out that problems can occur in implementation of the method of Cook et al. (2006) if proper account is not taken of correlation between samples when checking MCMC sampling algorithms. Difficulties can also arise from discretization when using sample based estimates of quantiles. Talts et al. (2018) suggest an alternative approach based on ranks and ways of adjusting for dependence, if that is an issue. They also show how to interpret the nature of any problems with the algorithm based on the kind of departure from uniformity observed. Closely related methods to those of Cook et al. (2006) have been applied for checking likelihood-free and variational inference algorithms (Wegmann et al., 2009, Prangle et al., 2014; Yao et al., 2017; Lee et al., 2019). In the context of likelihood-free inference 
using approximate Bayesian computation (ABC), these quantile-based checks have motivated recalibration algorithms to improve the quality of posterior approximations (Rodrigues et al. 2018). In these approaches, if simple importance sampling ABC computational methods are used, then repeated posterior approximations for different data can be done using the same prior samples, and the recalibration adjustments can be performed efficiently. Rodrigues et al. (2018) also note that their adjustments are feasible when fast approximations are available, and they discuss in detail calibration of inferences based on auxiliary models. Variational approximations can also benefit from the suggested adjustments.

One difficulty with the quantile-based adjustment methods mentioned above is that it seems difficult to apply them in the case of a multivariate $\theta$ when corrections in the dependence structure of the posterior are desired. In the next section we outline a novel alternative to the standard quantile-based inference adjustments. In particular, we consider applying the tower property of conditional expectation and law of total variance to relate prior and posterior means and covariances. When the posterior means and covariances are estimated by an approximate inference algorithm, we suggest adjustments so that the correct prior to posterior relationships hold after the adjustment.

After outlining the main idea in Section 2, Section 3 explains the detailed implementation in the case where particle approximations of the posterior distributions are available. Section 4 considers several applications involving likelihood-free inference, and checking and adjusting for approximate inferences in some deep neural network and Gaussian process regression models. Section 5 gives some concluding discussion.

\section{The basic idea}

Suppose that $\boldsymbol{\theta}$ is a possibly multivariate unknown of interest. We define a Bayesian model through a joint density $p(\boldsymbol{\theta}, \boldsymbol{y})=p(\boldsymbol{\theta}) p(\boldsymbol{y} \mid \boldsymbol{\theta})=p(\boldsymbol{y}) p(\boldsymbol{\theta} \mid \boldsymbol{y})$. We assume that the first two moments of the prior distribution exist. The tower property of conditional expectation allows us to write the prior mean as

$$
E(\boldsymbol{\theta})=E(E(\boldsymbol{\theta} \mid \boldsymbol{Y})),
$$

for $\boldsymbol{Y} \sim p(\boldsymbol{y})$. Now suppose some approximate inference algorithm is available, approximating the true posterior density $p(\boldsymbol{\theta} \mid \boldsymbol{y})$ by $\tilde{p}(\boldsymbol{\theta} \mid \boldsymbol{y})$, with approximate posterior mean and posterior

covariance matrix $\widetilde{E}(\boldsymbol{\theta} \mid \boldsymbol{y})$ and $\widetilde{\operatorname{Cov}}(\boldsymbol{\theta} \mid \boldsymbol{y})$ respectively. If our approximate inference algorithm is accurate, then

$$
E(\boldsymbol{\theta}) \approx E(\widetilde{E}(\boldsymbol{\theta} \mid \boldsymbol{Y}))
$$


Similarly, applying the law of total variance to the prior covariance matrix $\operatorname{Cov}(\boldsymbol{\theta})$ of $\boldsymbol{\theta}$,

$$
\operatorname{Cov}(\boldsymbol{\theta})=E(\operatorname{Cov}(\boldsymbol{\theta} \mid \boldsymbol{Y}))+\operatorname{Cov}(E(\boldsymbol{\theta} \mid \boldsymbol{Y}))
$$

for $\boldsymbol{Y} \sim p(\boldsymbol{y})$. Once again, if our approximate inference algorithm is accurate,

$$
\operatorname{Cov}(\boldsymbol{\theta}) \approx E(\widetilde{\operatorname{Cov}}(\boldsymbol{\theta} \mid \boldsymbol{Y}))+\operatorname{Cov}(\widetilde{E}(\boldsymbol{\theta} \mid \boldsymbol{Y}))
$$

for $\boldsymbol{Y} \sim p(\boldsymbol{y})$. The main idea of our checking and adjustment method is that given independent samples $\left(\boldsymbol{\theta}^{(i)}, \boldsymbol{y}^{(i)}\right) \sim p(\boldsymbol{\theta}) p(\boldsymbol{y} \mid \boldsymbol{\theta}), i=1, \ldots, I$, we can estimate the left-hand sides of (2) and $(4)$ based on the prior samples $\boldsymbol{\theta}^{(i)}$ (if the prior mean and covariance are not available analytically), and the right-hand sides of (2) and (4) based on the samples $\boldsymbol{y}^{(i)}$ and the approximations $\widetilde{E}\left(\boldsymbol{\theta} \mid \boldsymbol{y}^{(i)}\right)$ and $\widetilde{\operatorname{Cov}}\left(\boldsymbol{\theta} \mid \boldsymbol{y}^{(i)}\right)$. For checking, we can assess whether departures from equality in (2) and (4) are of practical concern. For inference adjustments, we can alter $\tilde{p}\left(\boldsymbol{\theta} \mid \boldsymbol{y}^{(i)}\right)$ so that exact equality holds in (2) and (4) for $\widetilde{E}\left(\boldsymbol{\theta} \mid \boldsymbol{y}^{(i)}\right)$ and $\widetilde{\operatorname{Cov}}\left(\boldsymbol{\theta} \mid \boldsymbol{y}^{(i)}\right)$ after adjustment. Although this is the basic idea, some further refinements are outlined below. Checking Bayesian inferences according to equation (2) is somewhat related to the idea of calibration of posterior means discussed in Gelman et al. (2013), p. 128, although the use of (2) and (4) jointly to check and adjust approximate inference methods is novel as far as we know. It may also be possible to extend to adjustments beyond second order moments using the law of total cumulance (Brillinger, 1969), but we do not consider this further.

\subsection{Use of a conditioning set}

A first observation (related to a similar remark in Prangle et al. (2014) for their quantile based diagnostic for likelihood-free inference algorithms) is that while achieving approximate equality in (2) and (4) is necessary for an approximate inference algorithm to be good, it is not sufficient. Consider, for example, the approximate inference method which always returns the prior as the estimated posterior. Then $E(\widetilde{E}(\boldsymbol{\theta} \mid \boldsymbol{Y}))=E(E(\boldsymbol{\theta}))=E(\boldsymbol{\theta})$, and $\operatorname{Cov}(\boldsymbol{\theta})=E(\widetilde{\operatorname{Cov}}(\boldsymbol{\theta} \mid \boldsymbol{Y}))+\operatorname{Cov}(\widetilde{E}(\boldsymbol{\theta} \mid \boldsymbol{Y}))=E(\operatorname{Cov}(\boldsymbol{\theta}))+\operatorname{Cov}(E(\boldsymbol{\theta}))=\operatorname{Cov}(\boldsymbol{\theta})$ so (2) and (4) hold exactly if we always estimate the posterior regardless of $\boldsymbol{y}$ by the prior. On the other hand, it is not hard to see that diagnosing the quality of the approximation using (2) and(4) could be useful. For example, suppose that our approximate inference algorithm always produces exact posterior mean values $E(\boldsymbol{\theta} \mid \boldsymbol{Y})=\widetilde{E}(\boldsymbol{\theta} \mid \boldsymbol{Y})$ while underestimating variability $\operatorname{Cov}(\boldsymbol{\theta} \mid \boldsymbol{Y}) \geq \widetilde{\operatorname{Cov}}(\boldsymbol{\theta} \mid \boldsymbol{Y}))$ where $\boldsymbol{A} \geq \boldsymbol{B}$ for covariance matrices $\boldsymbol{A}$ and $\boldsymbol{B}$ means that $\boldsymbol{A}-\boldsymbol{B}$ is non-negative definite. Then (2) would hold, but

$$
\operatorname{Cov}(\boldsymbol{\theta})=E(\operatorname{Cov}(\boldsymbol{\theta} \mid \boldsymbol{Y}))+\operatorname{Cov}(E(\boldsymbol{\theta} \mid \boldsymbol{Y})) \geq E(\widetilde{\operatorname{Cov}}(\boldsymbol{\theta} \mid \boldsymbol{Y}))+\operatorname{Cov}(\widetilde{E}(\boldsymbol{\theta} \mid \boldsymbol{Y})),
$$


and the situation we have considered is not unrealistic for some variational inference algorithms where point estimation may be excellent but posterior variability is substantially underestimated.

In the case of checking and adjustment of a posterior density $p\left(\boldsymbol{\theta} \mid \boldsymbol{y}_{\text {obs }}\right)$ for observed value $\boldsymbol{y}_{\text {obs }}$ of $\boldsymbol{y}$, it seems sensible to consider (again following Prangle et al. (2014)) a conditioning on $\boldsymbol{y} \in F$ in the joint model for some set $F$ with $\boldsymbol{y}_{\text {obs }} \in F$ in order to make the adjustment more relevant to the data observed. This will also avoid problems such as the one mentioned above where an approximate inference algorithm returning the prior would be considered satisfactory or in no need of adjustment. This is because after modifying equations (2) and (4) by conditioning on $\boldsymbol{y} \in F$, these modified relations will not hold, in general, for an approximate inference algorithm estimating the posterior density by the prior. The choice of $F$ will be discussed later in the examples. More explicitly, consider the joint model

$$
p^{F}(\boldsymbol{\theta}, \boldsymbol{y}) \propto p(\boldsymbol{\theta}) p(\boldsymbol{y} \mid \boldsymbol{\theta}) \mathbf{1}(\boldsymbol{y} \in F),
$$

where $\mathbf{1}(\cdot)$ denotes the indicator function, and samples from this joint model can be obtained by drawing $\left(\boldsymbol{\theta}^{\prime}, \boldsymbol{y}^{\prime}\right) \sim p(\boldsymbol{\theta}) p(\boldsymbol{y} \mid \boldsymbol{\theta})$ until $\boldsymbol{y}^{\prime} \in F$. Write $p^{F}(\boldsymbol{y})$ for the $\boldsymbol{y}$ marginal of $p^{F}(\boldsymbol{\theta}, \boldsymbol{y})$. In the model $p^{F}(\boldsymbol{\theta}, \boldsymbol{y})$ we can consider the following analogues of $(1)$ and $(3)$ :

$$
E(\boldsymbol{\theta} \mid \boldsymbol{Y} \in F)=E(E(\boldsymbol{\theta} \mid \boldsymbol{Y}) \mid \boldsymbol{Y} \in F),
$$

and

$$
\operatorname{Cov}(\boldsymbol{\theta} \mid \boldsymbol{Y} \in F)=E(\operatorname{Cov}(\boldsymbol{\theta} \mid \boldsymbol{Y}) \mid \boldsymbol{Y} \in F)+\operatorname{Cov}(E(\boldsymbol{\theta} \mid \boldsymbol{Y}) \mid \boldsymbol{Y} \in F),
$$

and then checking and adjustment can be based on assessment of departures from equality in (5) and 6) when $E(\boldsymbol{\theta} \mid \boldsymbol{Y})$ and $\operatorname{Cov}(\boldsymbol{\theta} \mid \boldsymbol{Y})$ are replaced by $\widetilde{E}(\boldsymbol{\theta} \mid \boldsymbol{Y})$ and $\widetilde{\operatorname{Cov}}(\boldsymbol{\theta} \mid \boldsymbol{Y})$. If our approximation is accurate, then

$$
E(\boldsymbol{\theta} \mid \boldsymbol{Y} \in F) \approx E(\widetilde{E}(\boldsymbol{\theta} \mid \boldsymbol{Y}) \mid \boldsymbol{Y} \in F),
$$

and

$$
\operatorname{Cov}(\boldsymbol{\theta} \mid \boldsymbol{Y} \in F) \approx E(\widetilde{\operatorname{Cov}}(\boldsymbol{\theta} \mid \boldsymbol{Y}) \mid \boldsymbol{Y} \in F)+\operatorname{Cov}(\widetilde{E}(\boldsymbol{\theta} \mid \boldsymbol{Y}) \mid \boldsymbol{Y} \in F)
$$

for samples from $p^{F}(\boldsymbol{\theta}, \boldsymbol{y})$. In the next section we describe in detail how checking and adjustment are done when approximate posterior distributions are given as particle approximations in the form of Monte Carlo samples. 


\section{Checking and adjustment strategy}

For an approximate inference algorithm leading to approximate posterior distributions $\tilde{p}(\boldsymbol{\theta} \mid \boldsymbol{Y})$ for $\boldsymbol{Y} \sim p^{F}(\boldsymbol{y})$ we have suggested checking and adjustment based on assessing departure from equality in (7) and (8). For data $\boldsymbol{y}$ we assume that the approximation $\tilde{p}(\boldsymbol{\theta} \mid \boldsymbol{y})$ comes in the form of an approximate posterior sample, $\boldsymbol{\theta}^{(1)}(\boldsymbol{y}), \ldots, \boldsymbol{\theta}^{(S)}(\boldsymbol{y})$, and if this is not the case, we assume that it is easy to generate from $\tilde{p}(\boldsymbol{\theta} \mid \boldsymbol{y})$ to obtain such a sample. Let $\left(\boldsymbol{\theta}^{(i)}, \boldsymbol{y}^{(i)}\right)$, $i=1, \ldots I$ denote independent draws from $p^{F}(\boldsymbol{\theta}, \boldsymbol{y})$.

Let us write

$$
\boldsymbol{\mu}^{L}=\frac{1}{I} \sum_{i=1}^{I} \boldsymbol{\theta}^{(i)} \quad \text { and } \quad \boldsymbol{\mu}^{R}=\frac{1}{I} \sum_{i=1}^{I} \boldsymbol{\mu}^{R}\left(\boldsymbol{y}^{(i)}\right)
$$

with

$$
\boldsymbol{\mu}^{R}\left(\boldsymbol{y}^{(i)}\right)=\frac{1}{S} \sum_{s=1}^{S} \boldsymbol{\theta}^{(s)}\left(\boldsymbol{y}^{(i)}\right)
$$

Here $\boldsymbol{\mu}^{L}$ and $\boldsymbol{\mu}^{R}$ are sample based estimates of the left- and right-hand sides of (7). Also write

$$
\boldsymbol{\Sigma}^{L}=\frac{1}{I-1} \sum_{i=1}^{I}\left(\boldsymbol{\theta}^{(i)}-\boldsymbol{\mu}^{L}\right)\left(\boldsymbol{\theta}^{(i)}-\boldsymbol{\mu}^{L}\right)^{T}
$$

and

$$
\Sigma^{R}=\Sigma^{R 1}+\Sigma^{R 2}
$$

where

$$
\begin{gathered}
\boldsymbol{\Sigma}^{R 1}=\frac{1}{I} \sum_{i=1}^{I} \boldsymbol{\Sigma}^{R 1}\left(\boldsymbol{y}^{(i)}\right), \\
\boldsymbol{\Sigma}^{R 1}\left(\boldsymbol{y}^{(i)}\right)=\frac{1}{S-1} \sum_{s=1}^{S}\left(\boldsymbol{\theta}^{(s)}\left(\boldsymbol{y}^{(i)}\right)-\boldsymbol{\mu}^{R}\left(\boldsymbol{y}^{(i)}\right)\right)\left(\boldsymbol{\theta}^{(s)}\left(\boldsymbol{y}^{(i)}\right)-\boldsymbol{\mu}^{R}\left(\boldsymbol{y}^{(i)}\right)\right)^{T},
\end{gathered}
$$

and

$$
\boldsymbol{\Sigma}^{R 2}=\frac{1}{I-1} \sum_{i=1}^{I}\left(\boldsymbol{\mu}^{R}\left(\boldsymbol{y}^{(i)}\right)-\boldsymbol{\mu}^{R}\right)\left(\boldsymbol{\mu}^{R}\left(\boldsymbol{y}^{(i)}\right)-\boldsymbol{\mu}^{R}\right)^{T}
$$

Here $\boldsymbol{\Sigma}^{L}$ and $\boldsymbol{\Sigma}^{R}$ are sample estimates of the left- and right-hand sides of $(8)$, with $\boldsymbol{\Sigma}^{R 1}$ and $\Sigma^{R 2}$ sample estimates of the first and second terms on the right-hand side of $(8)$. In the case 
where $\widetilde{E}\left(\boldsymbol{\theta} \mid \boldsymbol{y}^{(i)}\right)$ and $\widetilde{\operatorname{Cov}}\left(\boldsymbol{\theta} \mid \boldsymbol{y}^{(i)}\right)$ are known exactly, these can be substituted for $\boldsymbol{\mu}^{R}\left(\boldsymbol{y}^{(i)}\right)$ and $\boldsymbol{\Sigma}^{R}\left(\boldsymbol{y}^{(i)}\right)$ above. This can happen when the approximation is not in the form of a Monte Carlo sample but takes some analytical form such as for a variational approximation. With the above ideas and notation established, we consider next inference algorithm assessment, and then inference adjustments.

\subsection{Assessment of the inference algorithm}

The equalities $\boldsymbol{\mu}^{L}=\boldsymbol{\mu}^{R}$ and $\boldsymbol{\Sigma}^{L}=\boldsymbol{\Sigma}^{R}$ will not hold exactly. We suggest assessing whether components of $\boldsymbol{\mu}^{L}$ and $\boldsymbol{\mu}^{R}$ and of $\boldsymbol{\Sigma}^{L}$ and $\boldsymbol{\Sigma}^{R}$ differ by a large amount compared to the variability in their sample based estimation. The practical importance of the size of any difference for inferences and decisions should also be considered, but this question tends to be application specific. To assess variability in sample based estimation of $\boldsymbol{\mu}^{L}, \boldsymbol{\mu}^{R}, \boldsymbol{\Sigma}^{L}$ and $\boldsymbol{\Sigma}^{R}$ we suggest using the bootstrap. Consider resampling of the triples $\left(\boldsymbol{\theta}_{i}, \boldsymbol{\mu}^{R}\left(\boldsymbol{y}^{(i)}\right), \boldsymbol{\Sigma}^{R 1}\left(\boldsymbol{y}^{(i)}\right)\right)$, $i=1, \ldots, I$, with replacement, $B$ times. For each resample we compute $\boldsymbol{\mu}^{L}, \boldsymbol{\mu}^{R}, \boldsymbol{\Sigma}^{L}$ and $\boldsymbol{\Sigma}^{R}$ to obtain values $\boldsymbol{\mu}^{L b}, \boldsymbol{\mu}^{R b}, \boldsymbol{\Sigma}^{L b}, \boldsymbol{\Sigma}^{R b}, b=1, \ldots, B$. We can plot components of $\boldsymbol{\mu}^{L b}$ and $\boldsymbol{\mu}^{R b}$ against each other, as well as standard deviations and correlations derived from $\boldsymbol{\Sigma}^{L b}$ and $\boldsymbol{\Sigma}^{R b}$. Estimates $\boldsymbol{\mu}^{L}$ and $\boldsymbol{\Sigma}^{L}$ are direct estimates of the mean and covariance of $\boldsymbol{\theta}$ given $\boldsymbol{Y} \in F$ based on prior samples. Estimates $\boldsymbol{\mu}^{R}$ and $\boldsymbol{\Sigma}^{R}$ are indirect estimates based on posterior approximations. For assessment of an approximate inference algorithm, checks of location, scale and correlation are often directly meaningful to users. In contrast, marginal quantile based assessments require the user to back out information about the nature of any miscalibration in location and scale from histograms of $p$-values, which of course can be done but requires more sophistication. For our suggested plots of functions of $\boldsymbol{\mu}^{L B}, \boldsymbol{\mu}^{R b}$ and $\Sigma^{L b}, \Sigma^{R b}$ against each other, points lying above (below) the diagonal line indicate an average overestimation (underestimation) based on the posterior approximation. The use and meaning of these plots is discussed further in the examples of Section 4.

\subsection{Adjustment of the inference algorithm}

To adjust approximate inferences so that the equalities (7) and (8) hold, we can transform the particles $\boldsymbol{\theta}^{(s)}\left(\boldsymbol{y}^{(i)}\right)$. We assume that the components of $\boldsymbol{\theta}$ are unrestricted, which can be achieved by a preliminary transformation if necessary. Write $\boldsymbol{C}$ for the lower triangular Cholesky factor of $\boldsymbol{\Sigma}^{R 1}$. Also, assuming that $\boldsymbol{\Sigma}^{L}-\boldsymbol{\Sigma}^{R 2}$ is positive definite, write $\boldsymbol{T}$ for it's lower triangular Cholesky factor. Although $\Sigma^{L}-\Sigma^{R 2}$ is not guaranteed to be positive definite in general, it usually is, since the prior variation (captured by $\Sigma^{L}$ ) tends to be larger than the 
variability in the posterior means (estimated by $\Sigma^{R 2}$ ). We describe later how to handle the situation where $\boldsymbol{\Sigma}^{L}-\boldsymbol{\Sigma}^{R 2}$ is not positive definite, which can which can happen when there is a very poor approximation or because of the sampling variability in the estimation of $\boldsymbol{\Sigma}^{L}$ and $\Sigma^{R 2}$.

Now suppose we transform the samples $\boldsymbol{\theta}^{(s)}\left(\boldsymbol{y}^{(i)}\right), i=1, \ldots, I, s=1, \ldots, S$ to

$$
\tilde{\boldsymbol{\theta}}^{(s)}\left(\boldsymbol{y}^{(i)}\right)=\boldsymbol{\mu}^{L}+\left(\boldsymbol{\mu}^{R}\left(\boldsymbol{y}^{(i)}\right)-\boldsymbol{\mu}^{R}\right)+\boldsymbol{T} \boldsymbol{C}^{-1}\left(\boldsymbol{\theta}^{(s)}\left(\boldsymbol{y}^{(i)}\right)-\boldsymbol{\mu}^{R}\left(\boldsymbol{y}^{(i)}\right)\right) .
$$

The first two terms on the right-hand side of (9) perform a mean adjustment for the $i$ th replicate samples sufficient to ensure that (7) holds for the adjusted samples. The final term on the right-hand side of $(9)$ is a scaling of the mean-centred particles for the $i$ th replicate samples, and ensures that an empirical estimate of $E(\operatorname{Cov}(\theta \mid Y))$ based on the transformed particles leads to equality in (8). More precisely, denote the quantities $\boldsymbol{\mu}^{R}\left(\boldsymbol{y}^{(i)}\right), \boldsymbol{\mu}^{R}, \boldsymbol{\Sigma}^{R 1}\left(\boldsymbol{y}^{(i)}\right)$ etc. evaluated for the adjusted samples by $\tilde{\boldsymbol{\mu}}^{R}\left(\boldsymbol{y}^{(i)}\right), \tilde{\boldsymbol{\mu}}^{R}, \tilde{\boldsymbol{\Sigma}}^{R 1}\left(\boldsymbol{y}^{(i)}\right)$, etc. We have

$$
\tilde{\boldsymbol{\mu}}^{R}=\frac{1}{I} \sum_{i=1}^{I} \tilde{\boldsymbol{\mu}}^{R}\left(\boldsymbol{y}^{(i)}\right)
$$

with

$$
\tilde{\boldsymbol{\mu}}^{R}\left(\boldsymbol{y}^{(i)}\right)=\frac{1}{S} \sum_{s=1}^{S} \tilde{\boldsymbol{\theta}}^{(s)}\left(\boldsymbol{y}^{(i)}\right)=\boldsymbol{\mu}^{L}+\left(\boldsymbol{\mu}^{R}\left(\boldsymbol{y}^{(i)}\right)-\boldsymbol{\mu}^{R}\right),
$$

so that $\tilde{\boldsymbol{\mu}}^{R}=\boldsymbol{\mu}^{L}$ and (7) holds exactly for the adjusted samples. Next, note that

$$
\tilde{\boldsymbol{\mu}}^{R}\left(\boldsymbol{y}^{(i)}\right)-\tilde{\boldsymbol{\mu}}^{R}=\boldsymbol{\mu}^{R}\left(\boldsymbol{y}^{(i)}\right)-\boldsymbol{\mu}^{R}
$$

and hence $\tilde{\boldsymbol{\Sigma}}^{R 2}=\boldsymbol{\Sigma}^{R 2}$. Also, $\tilde{\boldsymbol{\theta}}^{(s)}\left(\boldsymbol{y}^{(i)}\right)-\tilde{\boldsymbol{\mu}}^{R}\left(\boldsymbol{y}^{(i)}\right)=\boldsymbol{T} \boldsymbol{C}^{-1}\left(\boldsymbol{\theta}^{(s)}\left(\boldsymbol{y}^{(i)}\right)-\boldsymbol{\mu}^{R}\left(\boldsymbol{y}^{(i)}\right)\right)$, and hence

$$
\tilde{\boldsymbol{\Sigma}}^{R 1}=\frac{1}{I} \sum_{i=1}^{I} \boldsymbol{T} \boldsymbol{C}^{-1} \boldsymbol{\Sigma}^{R 1}\left(\boldsymbol{y}^{(i)}\right) \boldsymbol{C}^{-\top} \boldsymbol{T}^{\top}=\boldsymbol{T} \boldsymbol{C}^{-1} \boldsymbol{\Sigma}^{R 1} \boldsymbol{C}^{-\top} \boldsymbol{T}^{\top}=\boldsymbol{T} \boldsymbol{T}^{\top}=\boldsymbol{\Sigma}^{L}-\boldsymbol{\Sigma}^{R 2}
$$

so that $\tilde{\boldsymbol{\Sigma}}^{R 1}=\boldsymbol{\Sigma}^{L}-\boldsymbol{\Sigma}^{R 2}$ and

$$
\tilde{\mathbf{\Sigma}}^{R}=\tilde{\mathbf{\Sigma}}^{R 1}+\tilde{\mathbf{\Sigma}}^{R 2}=\boldsymbol{\Sigma}^{L}-\boldsymbol{\Sigma}^{R 2}+\boldsymbol{\Sigma}^{R 2}=\boldsymbol{\Sigma}^{L}
$$

so (8) also holds exactly for the adjusted samples. Given samples $\boldsymbol{\theta}^{(s)}\left(\boldsymbol{y}_{\text {obs }}\right), s=1, \ldots, S$ for the observed data, we will transform them similarly to (9) to get an adjusted Monte Carlo sample from the posterior given $\boldsymbol{y}_{\text {obs }}$ to be used for inferential purposes:

$$
\tilde{\boldsymbol{\theta}}^{(s)}\left(\boldsymbol{y}_{\mathrm{obs}}\right)=\boldsymbol{\mu}^{L}+\left(\boldsymbol{\mu}^{R}\left(\boldsymbol{y}_{\mathrm{obs}}\right)-\boldsymbol{\mu}^{R}\right)+\boldsymbol{T} \boldsymbol{C}^{-1}\left(\boldsymbol{\theta}^{(s)}\left(\boldsymbol{y}_{\mathrm{obs}}\right)-\boldsymbol{\mu}^{R}\left(\boldsymbol{y}_{\mathrm{obs}}\right)\right) .
$$


As mentioned earlier it may happen that $\Sigma^{L}-\Sigma^{R 2}$ is not positive definite when the approximate inference algorithm gives particularly poor estimation of the posterior mean values, or due to sampling variability in the estimates $\Sigma^{L}$ and $\Sigma^{R 2}$. To handle this we can do a preliminary preprocessing in which $\boldsymbol{\theta}^{(s)}\left(\boldsymbol{y}^{(i)}\right)$ is changed to

$$
\boldsymbol{\mu}^{R}+\sqrt{\rho}\left(\boldsymbol{\mu}^{R}\left(\boldsymbol{y}^{(i)}\right)-\boldsymbol{\mu}^{R}\right)+\boldsymbol{\theta}^{(s)}\left(\boldsymbol{y}^{(i)}\right)-\boldsymbol{\mu}^{R}\left(\boldsymbol{y}^{(i)}\right),
$$

in which $\rho$ is a shrinkage parameter, $0<\rho<1$. It is easy to see that this results in shrinkage of the $\boldsymbol{\mu}^{R}\left(\boldsymbol{y}^{(i)}\right)$ for the new particles towards $\boldsymbol{\mu}^{R}$, with $\boldsymbol{\Sigma}^{R 2}$ changing to $\rho \boldsymbol{\Sigma}^{R 2}$. The preprocessing leaves $\boldsymbol{\mu}^{R}, \boldsymbol{\Sigma}^{R 1}\left(\boldsymbol{y}^{(i)}\right)$ and $\boldsymbol{\Sigma}^{R 1}$ unchanged. The parameter $\rho$ is chosen so that $\boldsymbol{\Sigma}^{L}-\rho \boldsymbol{\Sigma}^{R 2}$ is

positive definite. We suggest choosing it so that the smallest eigenvalue of $\boldsymbol{\Sigma}^{L}-\rho \boldsymbol{\Sigma}^{R 2}$ is equal to the smallest eigenvalue of $\Sigma^{R 1}$. Once this preprocessing is done if necessary, our adjustment can proceed as above.

The adjustment suggested (and the similar quantile based adjustment and checking methods in the literature) are computationally intensive, since they require repeated approximations to the posterior for different datasets. However, these approximations are sometimes easy to obtain. For example, in the case of approximate Bayesian computation methods using samples from the prior, the same prior samples can be reused for the approximation for different data. In the case where fast enough posterior approximations are available Rodrigues et al. (2018) point out that recalibration adjustments can still be attractive. The computations involved in recalibration are trivially parallelizable, consisting of independent computations for different datasets.

\section{Applications}

We consider three examples. The first concerns a likelihood-free inference application discussed in Rodrigues et al. (2018), and we compare our own adjustment method with the quantile-based method considered in their paper. Our second example involves inference in a deep neural network generalized linear mixed model (Tran et al., 2019). In this example our method corrects for empirical Bayes and variational computational approximations in approximate Bayesian inference. We also explain the features of this example which make the adjustment of Rodrigues et al. (2018) performs poorly compared to our new method. The third example considers predictive inference in a Gaussian process model, when a deep neural network regression is used as a surrogate for the Gaussian process. The inference adjustment is intended to make the deep neural network regression uncertainty quantification closer to that provided by the Gaussian process. This can be of interest because the Gaussian process computation can be intractable for large datasets. 


\subsection{Likelihood-free inference using an auxiliary model}

Our first example was considered in Rodrigues et al. (2018), where they considered a simple model that is useful in some finance and insurance applications. Consider $n$ independently and identically distributed observations $\boldsymbol{y}=\left(y_{1}, \ldots, y_{n}\right)$ of a random variable $Y$ that is defined as $Y=\sum_{k=1}^{\kappa} W_{k}$ where the $W_{k}$ are independent log-normal, $W_{k} \sim \log \operatorname{Normal}\left(\mu, \sigma^{2}\right)$. The density of $Y$ is difficult to compute, and Rodrigues et al. (2018) consider the so-called FentonWilkinson approximation (Fenton, 1960; Asmussen and Rojas-Nandayapa, 2008) which uses a $\log$-normal density matching the mean and variance of $Y$ to approximate the intractable density. $Y$ is approximated by $U \sim \operatorname{LogNormal}\left(m, s^{2}\right)$, where

$$
\begin{aligned}
& m=\mu+\log \kappa+\frac{1}{2}\left(\sigma^{2}-s^{2}\right) \\
& s^{2}=\log \left\{\left(\exp \left(\sigma^{2}\right)-1\right) / \kappa+1\right\} .
\end{aligned}
$$

As before, the observed $\boldsymbol{y}$ is denoted $\boldsymbol{y}_{\text {obs }}$. As a fast auxiliary model approximation to the posterior density for the parameter $\boldsymbol{\theta}=\left(\mu, \eta=\log \sigma^{2}\right)$, Rodrigues et al. (2018) consider a Laplace approximation when the likelihood is approximated by the Fenton-Wilkinson method. For data $\boldsymbol{y}$, write $\tilde{p}(\boldsymbol{y} \mid \boldsymbol{\theta})$ for the approximate likelihood, $\hat{\boldsymbol{\theta}}(\boldsymbol{y})=(\hat{\mu}(\boldsymbol{y}), \hat{\eta}(\boldsymbol{y}))$ for the mode of $\log h(\boldsymbol{\theta})$ where $h(\boldsymbol{\theta})=p(\boldsymbol{\theta}) \tilde{p}(\boldsymbol{y} \mid \boldsymbol{\theta})$, and $H(\hat{\boldsymbol{\theta}}(\boldsymbol{y}))$ for the Hessian of $-\log h(\boldsymbol{\theta})$ evaluated at $\hat{\boldsymbol{\theta}}(\boldsymbol{y})$. The posterior approximation based on the auxiliary Fenton-Wilkinson model and Laplace approximation is $N\left(\hat{\boldsymbol{\theta}}(\boldsymbol{y}), H(\hat{\boldsymbol{\theta}}(\boldsymbol{y}))^{-1}\right)$. Computation of this approximation is very fast, whereas an $\mathrm{ABC}$ approximation using $\hat{\boldsymbol{\theta}}(\boldsymbol{y})$ as the summary statistic is computationally demanding. After obtaining a posterior approximation for $\boldsymbol{\theta}$, we transform back to an approximation for $(\mu, \sigma)$. Although this example is concerned with likelihood-free inference, the use of an auxiliary model is not the traditional $\mathrm{ABC}$ approach commonly used in such settings. For $\mathrm{ABC}$, there are a number of other adjustment methods in the literature. These include methods based on regression (Beaumont et al., 2002; Blum, 2010; Blum and Tran, 2010; Blum and François, 2010) and on making adjustments to low-dimensional marginals (Nott et al. 2014 Li et al., 2017).

Rodrigues et al. (2018) consider simulating $n=10$ observations with $\kappa=10$ and $(\mu, \sigma)=$ $(0,1)$. The prior densities on $\mu$ and $\sigma$ are independent $N(0,1)$ and Gamma $(1,1)$ respectively. The recalibration procedure in Algorithm 2 of Rodrigues et al. (2018) incorporates kernel weights. With a uniform kernel, this corresponds to choosing a conditioning set $F$ in their approach, similar to that considered in Section 2, based on the support of the kernel centred on $\hat{\boldsymbol{\theta}}\left(\boldsymbol{y}_{\text {obs }}\right)$. In this example they use kernel bandwidth $h=\infty$, so that there is no reweighting or conditioning. As starting point for their adjustment, they generate a training sample of 10,000 parameter and data pairs from the prior. For each of these they compute their 
auxiliary model posterior approximation. Our own multivariate adjustment approach will be implemented based on these same posterior approximations. For our new method, we use a conditioning set $F$ of the form

$$
F=\left\{y: w_{1}^{-2}\left(\hat{\mu}(\boldsymbol{y})-\hat{\mu}\left(\boldsymbol{y}_{\mathrm{obs}}\right)\right)^{2}+w_{2}^{-2}\left(\hat{\eta}(\boldsymbol{y})-\hat{\eta}\left(\boldsymbol{y}_{\mathrm{obs}}\right)\right)^{2}<c\right\}
$$

where $w_{1}$ and $w_{2}$ are the prior predictive mean absolute deviations of $\hat{\mu}(\boldsymbol{y})$ and $\hat{\sigma}(\boldsymbol{y})$ respectively estimated from the training sample, and $c$ is chosen so that 1,000 of the 10,000 prior training samples are covered by $F$.

First, we consider a check on the quality of the approximate inferences provided by the auxiliary model. Figure 1 shows plots of the kind suggested in Section 3.1 of the components of bootstrap replicates of $\boldsymbol{\mu}^{L b}$ and $\boldsymbol{\mu}^{R b}$ against each other, and of posterior standard deviations

and correlations derived from bootstrap replicates of $\boldsymbol{\Sigma}^{L b}$ and $\boldsymbol{\Sigma}^{R b}$. The values for $\boldsymbol{\mu}^{L}$ and $\boldsymbol{\Sigma}^{L}$ are direct estimates of moments for $\boldsymbol{\theta}$ based on prior samples - the values for $\boldsymbol{\mu}^{R}$ and $\boldsymbol{\Sigma}^{R}$ are indirect estimates of moments based on the posterior approximation. The plots suggest some overall overestimation of posterior means for $\mu$, underestimation of posterior means for $\sigma$, underestimation of standard deviations for $\mu$ and $\sigma$, and overestimation of correlation between $\mu$ and $\sigma$.

The method of Rodrigues et al. (2018) works well in this example. The top panel of Figure 2 is similar to Figure 1 (b) in Rodrigues et al. (2018) and shows the estimated joint posterior based on the adjusted samples for their method, compared to a "gold standard" ABC method with small tolerance (see Rodrigues et al. (2018) for further details). The filled contours show the unadjusted auxiliary approximation. Shown in the bottom panel of Figure 2 is the adjusted posterior density estimate for our new approach, again comapred with the "gold standard" ABC analysis and the unadjusted approximation. Since the new method performs only mean and scale adjustments, the estimated joint posterior for $\boldsymbol{\theta}$ will be normal after adjustment when the auxiliary model posterior approximation is normal. Figure 2 shows that both methods manage to correct for the miscalibration of mean and scale in the initial auxiliary model approximation. The method of Rodrigues et al. (2018) works best here, but we explain in the next example why it sometimes performs badly.

\subsection{Variational and empirical Bayes approximations within a deep longitudinal mixed model}

Tran et al. (2019) describe generalized linear mixed models (GLMMs) for longitudinal data using deep neural network (DNN) basis functions. We consider inference for random effects variances in such models. Write $y_{j, t}$ for a binary response for an individual $j$ at time $t$, 

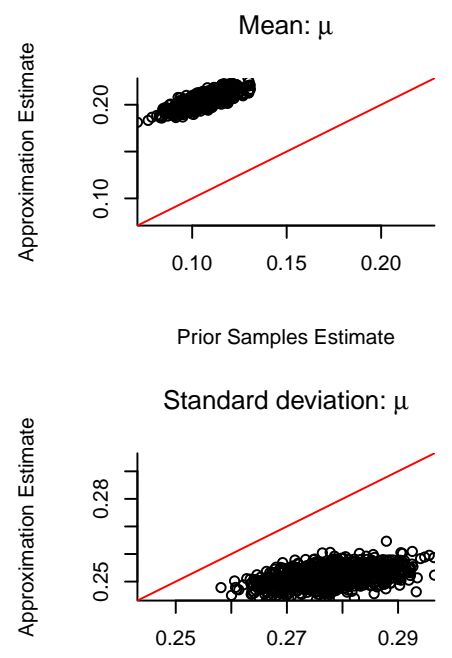

Prior Samples Estimate
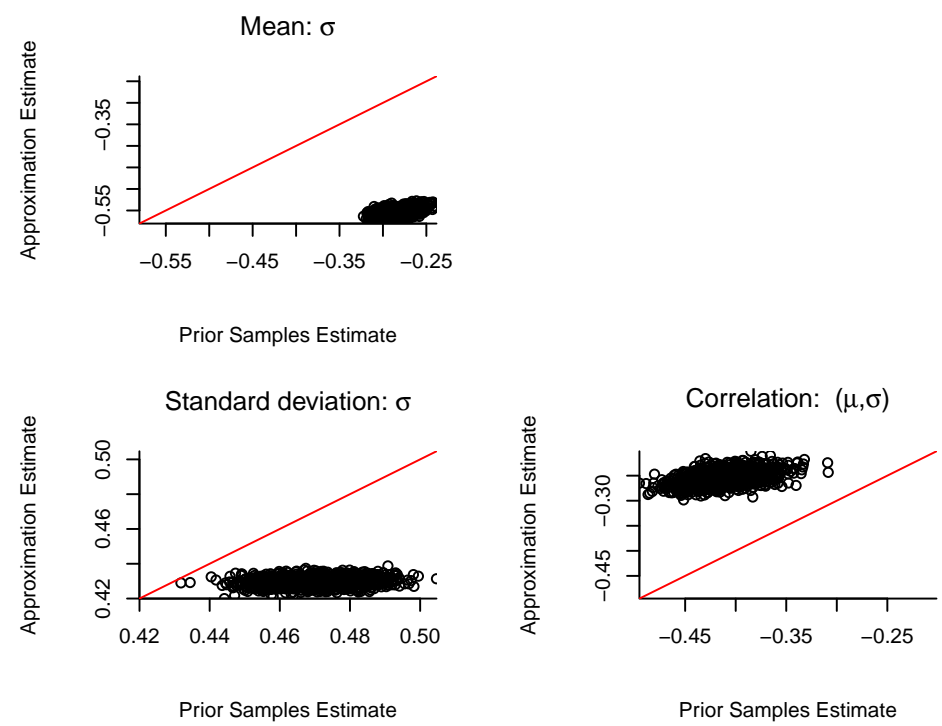

Figure 1: Plots of bootstrap replicates of components of $\boldsymbol{\mu}^{L}$ and $\boldsymbol{\mu}^{R}$ and of standard deviations and correlations derived from $\boldsymbol{\Sigma}^{L}$ and $\boldsymbol{\Sigma}^{R}$ against each other, with $B=1000$. Points above the red diagonal line indicate an overall overestimation by the posterior approximation, points below the red diagonal line indicate an overall underestimation.

$j=1, \ldots, J, t=1, \ldots, T_{j}$, and write $\boldsymbol{x}_{j, t}$ for a corresponding vector of covariates. A logistic deep GLMM assumes

$$
y_{j, t} \sim \operatorname{Bernoulli}\left(p_{j, t}\right), \quad \log \left(\frac{p_{j, t}}{1-p_{j, t}}\right)=\mathfrak{N}\left(\boldsymbol{x}_{j, t}, \boldsymbol{w}, \boldsymbol{\beta}+\boldsymbol{\alpha}_{j}\right),
$$

where $\mathfrak{N}\left(\boldsymbol{x}_{j, t}, \boldsymbol{w}, \boldsymbol{\beta}+\boldsymbol{\alpha}_{j}\right)$ is the scalar output of a feed-forward neural network with inner weights $\boldsymbol{w}$ and output weights $\boldsymbol{\beta}+\boldsymbol{\alpha}_{j}$, for input $\boldsymbol{x}_{j, t}$. If $L$ is the number of nodes in the last hidden layer of the neural network, the dimension of $\boldsymbol{\beta}$ and each $\boldsymbol{\alpha}_{j}$ is $L+1$, after inclusion of an intercept term. The vector $\boldsymbol{\beta}$ contains fixed effects parameters and $\boldsymbol{\alpha}_{j}$ are random effects, modelled as $\boldsymbol{\alpha}_{j} \sim N(\mathbf{0}, \boldsymbol{\Gamma})$ where $\boldsymbol{\Gamma}$ is diagonal with diagonal entries $\Gamma_{i}, i=0, \ldots, L$, and $\Gamma_{0}$ is the variance of the random intercepts. In our later example $L=10$, and only a single hidden layer will be used in the neural network. The model parameters are $\boldsymbol{\theta}=\left(\boldsymbol{w}, \boldsymbol{\beta}, \Gamma_{0}, \ldots, \Gamma_{L}\right)^{\top}$. Semiparametric versions of the above model which are more interpretable and in which some of the covariates are not transformed can also be considered. See Tran et al. (2019) for further discussion of the model.

For the neural network weights we use a prior which is normal, $\boldsymbol{w} \sim N\left(\mathbf{0}, \frac{1}{\gamma} \boldsymbol{I}\right), \beta \sim$ $N\left(\mathbf{0}, \frac{1}{\gamma} \boldsymbol{I}\right)$, where in our later data analysis $\gamma=130$. The value $\gamma=130$ was chosen based on prior predictive simulations. The parameters $\Gamma_{i} i=0, \ldots, L$ are assumed to follow a 

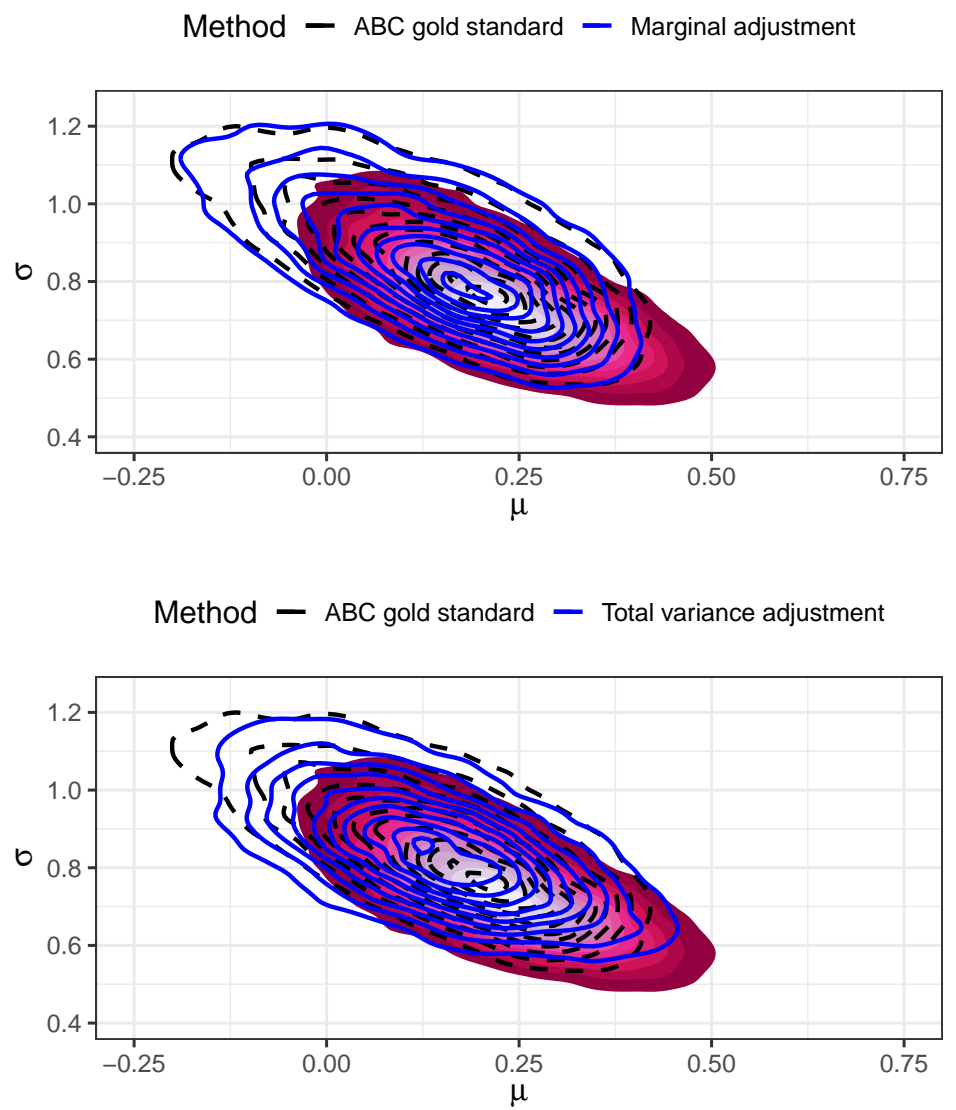

Figure 2: Contour plots of uncalibrated approximation, "gold standard" ABC approximation and adjusted approximations based on marginal calibration (top) and the new total variance calibration (bottom). All posterior approximations shown are two-dimensional kernel density estimates obtained from particle approximations to the posterior density for the different methods. 
prior in which they are independent with $\Gamma_{i} \sim \operatorname{Gamma}\left(a_{0}, b_{0}\right)$, but constrained to the region $\Gamma_{1}>\Gamma_{2}>\cdots>\Gamma_{L}$. This means that the basis functions are ordered according to the size of the corresponding random effect variance. We set $a_{0}=1$ and $b_{0}=10$ in this example, where the shape-scale parametrization of the gamma distribution is used here. We focus on inference for $\boldsymbol{\theta}=\left(\log \Gamma_{1}, \ldots, \log \Gamma_{L}\right)^{\top}$. It is challenging to make inferences about a large set of random effects variance parameters here unless the number of subjects is large. In generalized linear mixed models, maximum likelihood estimates or posterior mode estimates with weak priors often lead to degenerate estimates (Chung et al., 2015). Here we use strong shrinkage priors and a random effect for each basis function to define a predictive model which flexibly describes within subject dependence.

We use our adjustment method to approximate full Bayes inference using the normal prior on the weights above, based on the approximate normal posterior produced by the algorithm of Tran et al. (2019). Tran et al. (2019) approximate Bayesian inference under a different prior specification for the weights and using variational approximation methods and empirical Bayes methods for hyperparameter estimation. See Section 6.1.4 of their paper. Tran et al. (2019) do not impose the identification constraint $\Gamma_{1}>\ldots,>\Gamma_{L}$ for inference, and in their normal posterior approximation it is assumed that the covariance matrix has the low-rank plus diagonal form $\boldsymbol{B} \boldsymbol{B}^{\top}+\boldsymbol{D}^{2}$, where $\boldsymbol{D}$ is diagonal and it will be assumed here that $\boldsymbol{B}$ is a column vector. The approximate posterior samples needed for our adjustment method are obtained by simulating from the normal variational posterior, and then reordering components so that $\Gamma_{1}>\cdots>\Gamma_{L}$. We take the effect of the relabelling as another aspect of the approximation that our method aims to correct.

Similar to the previous example, we use a conditioning set $F$. If $\boldsymbol{y}$ is a simulated dataset from the prior with $\boldsymbol{y}=\left(\boldsymbol{y}_{1}^{\top}, \ldots, \boldsymbol{y}_{n}^{\top}\right)^{\top}, \boldsymbol{y}_{j}=\left(y_{j, 1}, \ldots, y_{j, T_{j}}\right)^{\top}$, then we use

$$
F=\left\{y: \operatorname{dist}\left(\boldsymbol{y}, \boldsymbol{y}_{\text {obs }}\right)<\epsilon\right\}
$$

where $\boldsymbol{y}_{\mathrm{obs}}$ is the observed data, $\boldsymbol{y}_{\mathrm{obs}}=\left(\boldsymbol{y}_{\mathrm{obs}, 1}^{\top}, \ldots, \boldsymbol{y}_{\mathrm{obs}, n}^{\top}\right)^{\top}, \boldsymbol{y}_{\mathrm{obs}, j}=\left(y_{\mathrm{obs}, j 1}, \ldots, y_{\mathrm{obs}, j T_{j}}\right)^{\top}$, $0<\epsilon<1$ is a tolerance parameter, and

$$
\operatorname{dist}\left(\boldsymbol{y}, \boldsymbol{y}_{\mathrm{obs}}\right)=\frac{1}{n} \sum_{j=1}^{n} \operatorname{dist}_{\mathrm{Jaccard}}\left(\boldsymbol{y}_{j}, \boldsymbol{y}_{\mathrm{obs}, j}\right),
$$

with dist $_{\text {Jaccard }}(\boldsymbol{u}, \boldsymbol{v})$ the Jaccard distance (Jaccard, 1901) between two binary vectors with the same length. If $a$ is the number of components where $\boldsymbol{u}$ and $\boldsymbol{v}$ are both 1 and $b$ is the number of components where $\boldsymbol{u}$ and $\boldsymbol{v}$ take different values, then $\operatorname{dist}_{\text {Jaccard }}(\boldsymbol{u}, \boldsymbol{v})=b /(a+b)$, where here by convention $0 / 0$ is defined to be 0 . We use our adjustment method with $I=200$ 

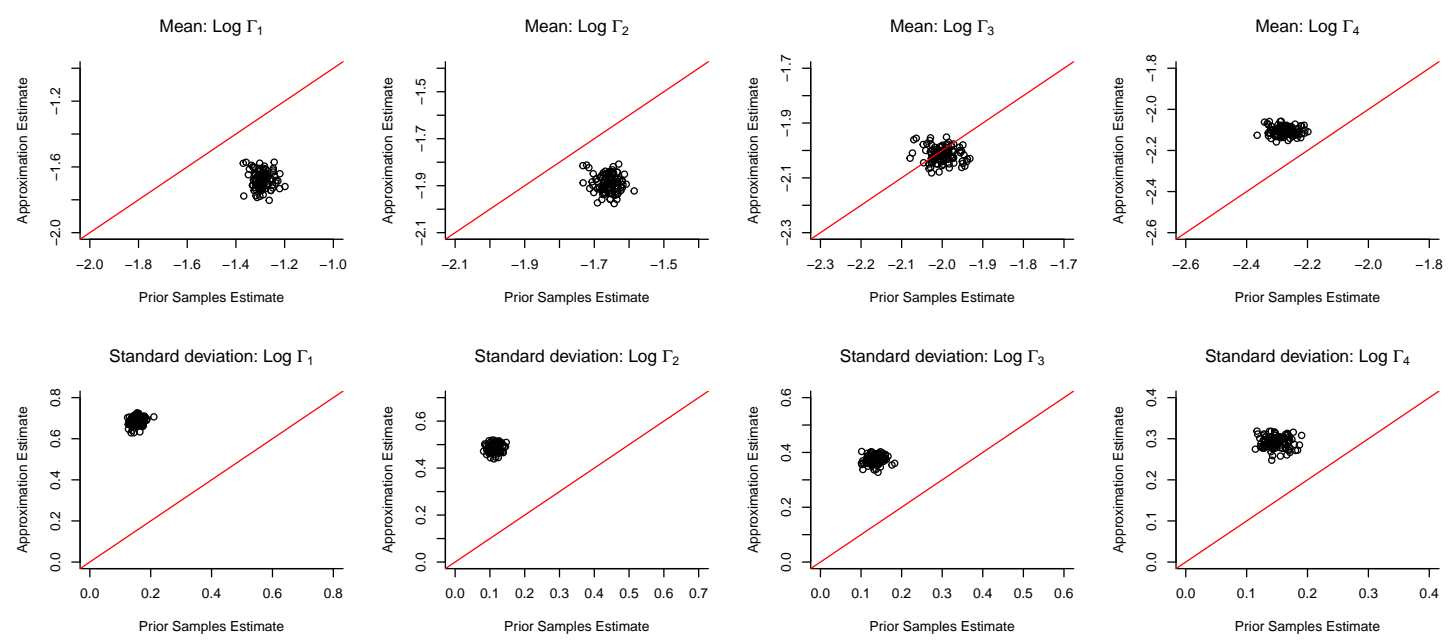

Figure 3: Plots of bootstrap replicates of components of $\boldsymbol{\mu}^{L}$ and $\boldsymbol{\mu}^{R}$ (top row) and of standard deviations derived from $\Sigma^{L}$ and $\Sigma^{R}$ (bottom row) against each other for $\left(\log \Gamma_{1}, \log \Gamma_{2}, \log \Gamma_{3}, \log \Gamma_{4}\right)$. Points above the diagonal line indicate an overall overestimation by the posterior approximation, points below the red diagonal line indicate an overall underestimation.

prior replicates, and choose $\epsilon$ in the conditioning set so that 100 of these prior replicates are kept for the adjustment.

We consider an analysis of a German health care dataset (Geil et al., 1997) consisting of yearly records of hospitalized status of 1887 German workers. The response $y_{j, t}=1$ if worker $j$ was hospitalized in year $t$, and $y_{j, t}=0$ otherwise. The covariates include gender, age, income, education, marriage status, job type and insurance type. There are 12 predictors in total after using dummy variables to represent the categorical variables. We use only the first 500 panels in the dataset, since fitting the deep GLMM model is computationally expensive.

\subsubsection{Assessment of approximate inference}

First, consider our bootstrap assessment plots for $\boldsymbol{\Delta}=\left(\Delta_{1}, \Delta_{2}, \Delta_{3}, \Delta_{4}\right)^{\top}$, where $\Delta_{j}=\log \Gamma_{j}$. We focus on the four largest random effects variance parameters since they are the most important, and summarizing the results graphically is easier with a smaller number of parameters. Figure 3 shows the plots for the mean (top row) and standard deviation (bottom row). We see that the approximate inference method underestimates the largest $\log \Gamma_{j}$ values, and overestimates their variability. 


\subsubsection{Approximate inference adjustments}

When the value of $E\left(\Delta_{j} \mid y^{(i)}\right)$ is estimated by $\hat{\Delta}_{j}^{(i)}$, the squared estimation error is $\left|\Delta_{j}^{(i)}-\hat{\Delta}_{j}^{(i)}\right|^{2}$ where $\Delta_{j}^{(i)}$ is the value of $\Delta_{j}$ used in simulating the data for replicate $i$. Figure 4 shows boxplots of these squared estimation errors for estimated posterior means obtained from unadjusted and adjusted posterior samples. The total variance adjustment improves substantially on the unadjusted method. We also tried the adjustment method of Rodrigues et al. (2018), which does not work well in this example (results not shown). The reason is that the unadjusted posterior approximation is very poor here, and the method of Rodrigues et al. (2018) obtains adjusted sample components by evaluating marginal posterior quantile functions conditional on the observed data at certain points. If the quantile functions are estimated based on the empirical distribution of a particle posterior estimate, then the adjusted samples cannot extend beyond the observed range of the unadjusted particles. Hence, if a good approximation requires putting posterior mass beyond the range of the unadjusted particles, the method of Rodrigues et al. (2018) can perform badly. The total variance method can still give useful results here because of the way that adjustments are based on mean and scale adjustments rather than quantiles.

Figure 5 shows, for the observed data, kernel estimates of the univariate and bivariate marginal posterior densities obtained with and without the total variance adjustment. Here we do not know the true parameter values. However, given the improvements demonstrated in Figure 4 for the simulated data, inference based on the adjusted approach seems preferable, and furthermore this adjustment leads to substantially different estimated posterior densities.

\subsection{Approximate Gaussian process regression predictive inference using a deep neural network surrogate}

Our final example considers application of a deep neural network regression model as a surrogate for Gaussian process regression predictive inference. We consider a $D=2$ dimensional input space. Suppose data $\left(\boldsymbol{z}_{\text {obs }}, \boldsymbol{X}_{\text {obs }}\right)$ are observed, where $\boldsymbol{z}_{\text {obs }}=\left(z_{\mathrm{obs}, 1}, \ldots, z_{\mathrm{obs}, n}\right)^{\top}$ is a vector of $n$ observed values for a response $\boldsymbol{z}$ and $\boldsymbol{X}_{\text {obs }}$ is a corresponding matrix of inputs with $n$ rows. The $i$ th row of $\boldsymbol{X}_{\mathrm{obs}}$ is denoted $\boldsymbol{x}_{\mathrm{obs}, i}$, and gives the value of the input $\boldsymbol{x}=\left(x_{1}, x_{2}\right)^{\top}$ for the response $\boldsymbol{z}_{\mathrm{obs}, i}$. We wish to make predictive inference for a future response $\boldsymbol{z}^{*}$ for input $\boldsymbol{x}^{*}$, and we will treat $\boldsymbol{x}$ as random for the purpose of deriving appropriate adjustments. The main purpose of this example is to show how our adjustment method applies in a setting which involves predictive inference. 

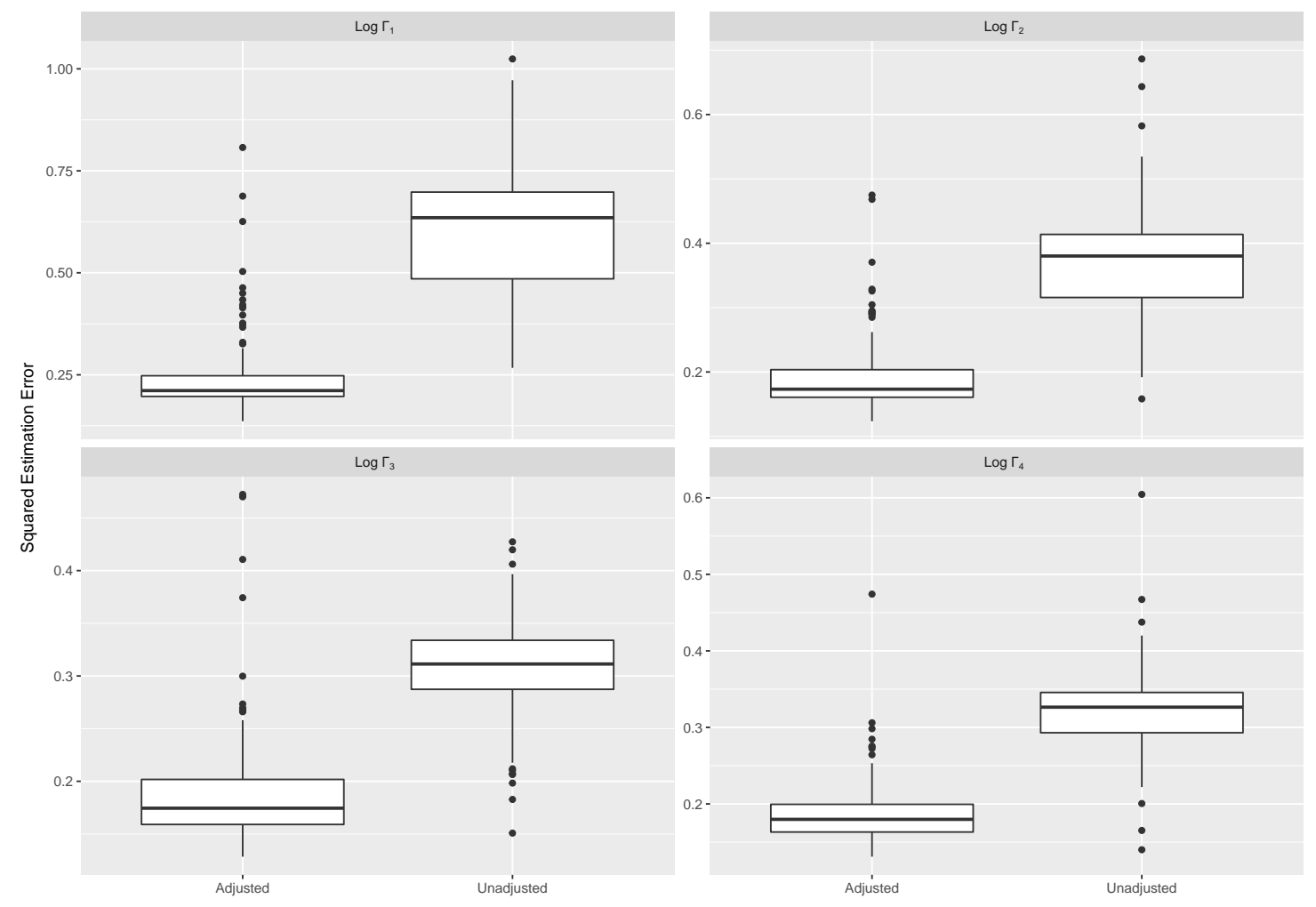

Figure 4: Boxplots of squared estimation errors for $\Delta_{j}=\log \Gamma_{j}, j=1, \ldots, 4$, with and without adjustment, treating each simulation replicate in turn as the observed data 

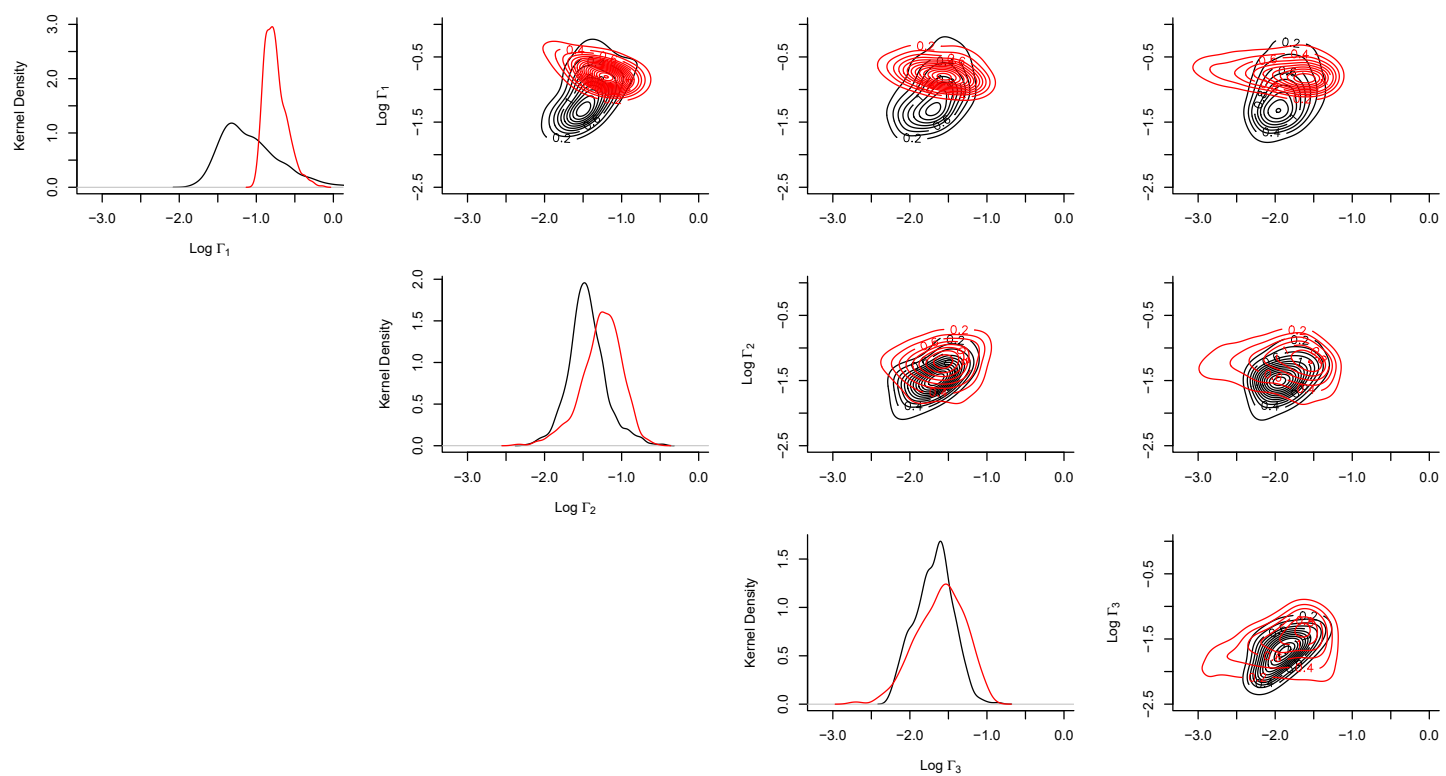

— Without Adjustment

With Adjustment

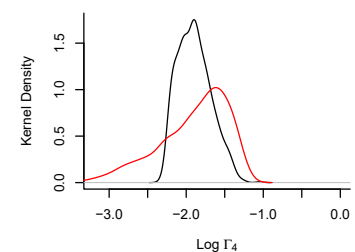

Figure 5: Univariate (diagonal panels) and bivariate (off-diagonal panels) estimated marginal posterior densities with (red) and without (black) total variance adjustment for $\log \Gamma_{j}, j=1, \ldots, 4$. 
For predictive inference adjustments following the framework of Section 3.2, we will start with $R$ simulated datasets from the prior, with each dataset containing training and test samples respectively,

$$
\left(z_{i}^{(r)}, \boldsymbol{x}_{i}^{(r)}\right), i=1, \ldots, n, \quad\left(z_{j}^{(*, r)}, \boldsymbol{x}_{j}^{(*, r)}\right), j=1, \ldots, m
$$

with $r=1, \ldots, R$ indexing the different replicates. The training and test inputs are generated independently as bivariate normal, $N\left(\mathbf{0}, \boldsymbol{\Sigma}_{x}\right)$ where the covariance matrix $\boldsymbol{\Sigma}_{x}$ has upper triangular Cholesky factor $\boldsymbol{R}_{x} . \boldsymbol{R}_{x}$ has non-zero elements $r_{k l}, k \geq l$ which we generated independently from an $N(0,0.5)$ density here. $\Sigma_{x}$ is the same for all the replicates. The responses are generated conditionally on inputs from a regression model,

$$
z_{i}^{(r)}=f^{(r)}\left(\boldsymbol{x}_{i}^{(r)}\right)+\epsilon_{i}^{(r)}, \quad z_{j}^{(*, r)}=f^{(r)}\left(\boldsymbol{x}_{j}^{(*, r)}\right)+\epsilon_{j}^{(*, r)}
$$

where $\epsilon_{i}^{(r)}, i=1, \ldots, n, \epsilon_{j}^{(*, r)}, j=1, \ldots, m$ are independent $N\left(0,\left(\sigma^{2}\right)^{(r)}\right)$ and $f^{(r)}(\cdot)$ is a mean function. The values $\left(\sigma^{2}\right)^{(r)}$ are simulated independently from a prior $p\left(\sigma^{2}\right)$.

The function $f^{(r)}(\cdot)$ is simulated from a Gaussian process prior, and we discuss this next. Write

$$
\begin{aligned}
\boldsymbol{X}^{(r)} & =\left(\boldsymbol{x}_{1}^{(r)}, \ldots, \boldsymbol{x}_{n}^{(r)}\right)^{\top}, \quad \boldsymbol{X}^{(*, r)}=\left(\boldsymbol{x}_{1}^{(*, r)}, \ldots, \boldsymbol{x}_{m}^{(*, r)}\right)^{\top}, \quad \boldsymbol{X}^{(+, r)}=\left[\boldsymbol{X}^{(r)^{\top}} \boldsymbol{X}^{(*, r)^{\top}}\right]^{\top}, \\
\boldsymbol{F}^{(r)} & =\left(f^{(r)}\left(x_{1}^{(r)}\right), \ldots, f^{(r)}\left(x_{n}^{(r)}\right)\right)^{\top}, \boldsymbol{F}^{(*, r)}=\left(f^{(r)}\left(x_{1}^{(*, r)}\right), \ldots, f^{(r)}\left(x_{m}^{(*, r)}\right)\right)^{\top}, \boldsymbol{F}^{(+, r)}=\left(\boldsymbol{F}^{(r)^{\top}}, \boldsymbol{F}^{(*, r)}\right)^{\top} \\
\boldsymbol{z}^{(r)} & =\left(z_{1}^{(r)}, \ldots, z_{n}^{(r)}\right)^{\top}, \quad \boldsymbol{z}^{(*, r)}=\left(z_{1}^{(*, r)}, \ldots, z_{m}^{(*, r)}\right)^{\top}, \quad \boldsymbol{z}^{(+, r)}=\left(\boldsymbol{z}^{(r)}, \boldsymbol{z}^{(*, r)^{\top}}\right)^{\top} .
\end{aligned}
$$

The required values for $f^{(r)}(\cdot)$ in $(11)$ are generated under the Gaussian process prior as

$$
\boldsymbol{F}^{(+, r)} \sim N\left(\mathbf{0}, \boldsymbol{C}\left(\boldsymbol{X}^{(+, r)}, \boldsymbol{X}^{(+, r)} ; \boldsymbol{\zeta}^{(r)}\right)\right.
$$

where $\boldsymbol{C}\left(\boldsymbol{X}^{(+, r)}, \boldsymbol{X}^{(+, r)} ; \boldsymbol{\zeta}\right)$ is the $(m+n) \times(m+n)$ covariance matrix with entry $(i, j)$ being $C\left(\boldsymbol{x}_{i}^{(+, r)}, \boldsymbol{x}_{j}^{(+, r)} ; \boldsymbol{\zeta}\right), \boldsymbol{x}_{l}^{(+, r)}$ denotes the $l$ th row of $\boldsymbol{X}^{(+, r)}$, and $C\left(\boldsymbol{x}, \boldsymbol{x}^{\prime} ; \boldsymbol{\zeta}\right)$ is the Matérn covariance function

$$
C\left(\boldsymbol{x}, \boldsymbol{x}^{\prime} ; \boldsymbol{\zeta}\right)=\tau^{2} \frac{2^{1-\nu}}{\Gamma(\nu)}\left(\sqrt{2 \nu} \frac{\left\|\boldsymbol{x}-\boldsymbol{x}^{\prime}\right\|}{\lambda}\right)^{\nu} K_{\nu}\left(\sqrt{2 \nu} \frac{\left\|\boldsymbol{x}-\boldsymbol{x}^{\prime}\right\|}{\lambda}\right),
$$

where $\left\|\boldsymbol{x}-\boldsymbol{x}^{\prime}\right\|$ is the Euclidean distance between $\boldsymbol{x}$ and $\boldsymbol{x}^{\prime}, \boldsymbol{\zeta}=\left(\tau^{2}, \lambda\right), K_{\nu}(\cdot)$ denotes the modified Bessel function of the second kind, and $\nu$ will be fixed at 1.5. For more background on Gaussian process regression see Rasmussen and Williams (2006) or Cressie and Wikle (2011).

Each $\boldsymbol{\zeta}^{(r)}, r=1, \ldots, R$, is simulated independently from a prior distribution in which $\tau^{2}$ and $\lambda$ are independent, $\tau^{2} \sim I G\left(a_{\tau}, b_{\tau}\right), \lambda \sim I G\left(a_{\lambda}, b_{\lambda}\right)$. Our prior for $\sigma^{2}$ is inverse gamma, $I G\left(a_{\sigma}, b_{\sigma}\right)$. Prior hyperparameters are $a_{\sigma}=3, b_{\sigma}=0.2, a_{\tau}=14.5, b_{\tau}=6.75, a_{\lambda}=b_{\lambda}=9$. 
This gives $E\left(\sigma^{2}\right)=0.1, \operatorname{Var}\left(\sigma^{2}\right)=0.01, E\left(\tau^{2}\right)=0.1, \operatorname{Var}\left(\tau^{2}\right)=0.02$ and assigns a fairly diffuse prior to the correlation between $f^{(r)}$ values at two inputs separated by unit Euclidean distance. We also simulate one further replicate for which the training set values take the role of the observed data $\left(\boldsymbol{z}_{\text {obs }}, \boldsymbol{X}_{\text {obs }}\right)$.

We approximate Gaussian process predictive inference using dense layer feed-forward neural network (FNN) regression models. This can be of interest since exact Gaussian process computations can be difficult for large $n$. There is a large literature on how to do Gaussian process computations efficiently for large datasets, and we make no effort to compare the adjustment described here with other methods for this task. The purpose of our example here is to illustrate the use of our adjustment method for predictive inference, where a surrogate model is being used which is more tractable than the original one. We consider an example with $n=1000$, since we wish to compare with exact Gaussian process answers. The neural network architecture we use has two hidden layers of size 64 each with ReLU activation functions followed by a univariate linear dense layer for the output. To avoid overfitting we employ $L 2$ regularization and a dropout rate of 0.3 at the hidden layers. The unadjusted deep learning regression model is estimated using the $\mathrm{R}$ package keras (Chollet and Allaire, 2018) and is denoted as unadjusted DNN below when discussing the results.

\subsubsection{Adjustment and choice of conditioning set}

Consider predictive inference on a yet to be observed response value $z^{*}$ at a known input value $\boldsymbol{x}^{*}$ based on the observed training data. In the notation of Section 3.2, we apply our adjustment to inference for $\theta=z^{*}$. We will use a Gaussian plug-in predictive density from the neural network at $\boldsymbol{x}^{*}$ as the posterior approximation for $z^{*}$ rather than some particle approximation. In this experiment one of the replicate datasets denoted $\boldsymbol{y}^{(i)}$ in Section 3.2 consists of a training dataset of responses and inputs of size $n,(\boldsymbol{z}, \boldsymbol{X})$ say, as well as a test input $\boldsymbol{x}^{*}$. The replicate datasets $\boldsymbol{y}^{(i)}$ retained by the conditioning set for our adjustment will take the form $\left(\boldsymbol{z}^{(r)}, \boldsymbol{X}^{(r)}\right), \boldsymbol{x}_{l}^{(*, r)}$ for some combinations of $r$ and $l$. A total of $I$ replicates will be retained and we describe how the corresponding $(r, l)$ pairs are obtained below. The replicates $\left(\theta^{(i)}, \boldsymbol{y}^{(i)}\right)$ used in the adjustment process will be dependent here if $m>1$, because some of the training sets are shared between different $\left(\theta^{(i)}, \boldsymbol{y}^{(i)}\right)$ replicates, as we simulate more than one test input per Gaussian process replicate. This does not complicate the estimation of means and covariances for our adjustment, but the construction in Section 3.1 of plots for assessment using the bootstrap would need to be modified to account for the dependence. This will not be considered further here, since we focus on predictive inference adjustments.

To describe the conditioning set used for our adjustment method, write $\hat{\tau}^{(r)}, \hat{\lambda}^{(r)}$ and $\hat{\sigma}^{(r)}$ 
for estimates of $\tau, \lambda$ and $\sigma$ for replicate $r, r=1, \ldots, R$. The corresponding values for the observed data are written simply as $\hat{\tau}, \hat{\lambda}, \hat{\sigma}$. Estimates of $\tau^{2}, \lambda$ are obtained based on weighted least squares fits to empirical variograms using the $\mathrm{R}$ package geoR (Ribeiro Jr. and Diggle, 2001), while the estimate for $\sigma^{2}$ is simply the residual variance in the training data. Write $\mathcal{S}=\mathcal{S}(\epsilon)$ for the set of indices of those replicates $r \in\{1, \ldots, R\}$ for which

$$
d\left((\boldsymbol{z}, \boldsymbol{X}),\left(\boldsymbol{z}_{\mathrm{obs}}, \boldsymbol{X}_{\mathrm{obs}}\right)\right)=w_{1}^{-2}\left(\hat{\tau}-\hat{\tau}^{(r)}\right)^{2}+w_{2}^{-2}\left(\hat{\lambda}-\hat{\lambda}^{(r)}\right)^{2}+w_{3}^{-2}\left(\hat{\sigma}-\hat{\sigma}^{(r)}\right)^{2}<\epsilon,
$$

where $\epsilon>0$ is a tolerance and the weights $w_{1}, w_{2}, w_{3}$ are the prior standard deviations of the estimates for $\tau, \lambda$ and $\sigma$ respectively across the $R$ Gaussian process replicates. We will choose $\epsilon$ so that $\mathcal{S}$ contains $S<R$ elements. Then $\mathcal{S}$ is the index set of the $S$ replicates with covariance hyperparameter estimates closest to the observed ones in a weighted Euclidean distance.

Next, consider the distances

$$
d_{j}^{(*, r)}=\min _{i=1, \ldots, n} \sqrt{\left(\boldsymbol{x}_{i}^{(r)}-\boldsymbol{x}_{j}^{(*, r)}\right)^{\top} \boldsymbol{\Sigma}_{x}^{-1}\left(\boldsymbol{x}_{i}^{(r)}-\boldsymbol{x}_{j}^{(*, r)}\right)}
$$

$j=1, \ldots, m, r=1, \ldots, I$. The value $d_{j}^{(*, r)}$ is the distance of $\boldsymbol{x}_{j}^{(*, r)}$ to the nearest training input in replicate $r$. We consider dividing these distances for the test inputs into a set of $K$ bins. Let

$$
\bar{d}^{r}=\frac{\max _{j, r}\left(d_{j}^{(*, r)}\right)-\min _{j, r}\left(d_{j}^{(*, r)}\right)}{K-1},
$$

and define the bins as $B_{k}=\left[c_{k}, d_{k}\right)$ for $k=0, \ldots, K-1$ where

$$
c_{k}=\frac{1}{R} \sum_{r=1}^{R}\left(\min _{j, r}\left(d_{j}^{(*, r)}\right)+\bar{d}^{r} k\right), \quad \frac{1}{R} \sum_{r=1}^{R}\left(\min _{j, r}\left(d_{j}^{(*, r)}\right)+\bar{d}^{r}(k+1)\right) .
$$

For any test input $\boldsymbol{x}^{*}$ and training set of inputs $\boldsymbol{X}$ write $k\left(\boldsymbol{x}^{*}, \boldsymbol{X}\right)$ for the index of the bin in which $\boldsymbol{x}^{*}$ lies for training inputs $\boldsymbol{X}$. For a simulated training set $(\boldsymbol{z}, \boldsymbol{X})$ from the prior and simulated test input $\boldsymbol{x}^{\prime}$, the conditioning set for our adjustment for predictive inference at $\boldsymbol{x}^{*}$ based on the observed training set is

$$
F\left(\boldsymbol{x}^{*}, \boldsymbol{X}\right)=\left\{(\boldsymbol{z}, \boldsymbol{X}), \boldsymbol{x}^{\prime}: d\left((\boldsymbol{z}, \boldsymbol{X}),\left(\boldsymbol{z}_{\text {obs }}, \boldsymbol{X}_{\text {obs }}\right)\right)<\epsilon, k\left(\boldsymbol{x}^{\prime}, \boldsymbol{X}\right)=k\left(\boldsymbol{x}^{*}, \boldsymbol{X}_{\text {obs }}\right)\right\}
$$

As discussed above, $\epsilon$ is chosen so that $\mathcal{S}$ has $S<R$ elements.

With input $\boldsymbol{x}^{*}$ for the observed training data, the above conditioning set results in using for the predictive adjustment the replicates $\boldsymbol{y}^{(i)}$ given by

$$
\left\{\left(\boldsymbol{z}^{(r)}, \boldsymbol{X}^{(r)}\right), \boldsymbol{x}_{l}^{(*, r)}: r \in \mathcal{S}, 1 \leq l \leq m, k\left(\boldsymbol{x}^{*}, \boldsymbol{X}_{\mathrm{obs}}\right)=k\left(\boldsymbol{x}_{l}^{(*, r)}, \boldsymbol{X}^{(r)}\right)\right\}
$$


Note that the replicates used in the adjustment, and the quantities $\boldsymbol{\mu}^{L}, \boldsymbol{\mu}^{R}, \boldsymbol{\Sigma}^{L}$ and $\boldsymbol{\Sigma}_{R}$ required for the adjustment, are the same for test inputs in the same bin for the observed data. So we compute adjustments that are bin specific, applying to all inputs in the same bin. The full adjustment algorithm is given in Algorithm 1. We used $R=1000$ prior replicates, $K=10$ bins, $n=1,000$ training samples per dataset, $m=100$ test samples per dataset, and $S=100$.

\subsubsection{Benchmarking and results}

To evaluate the performance of our adjustment, we compare the unadjusted DNN to our adjusted DNN inferences. Exact Gaussian process predictive inferences are also considered using the assumed Matérn covariance function fitted using the R package GPfit (MacDonald et al. 2015). Figure 6 shows boxplots of the unadjusted, adjusted and full GP inferences for the observed data test inputs where performance is evaluated according to a logarithmic scoring rule. For displaying the results, the distances of test set inputs to the nearest training input were partitioned into four distance intervals of equal length. The adjustment procedure improves predictive inference from the neural network surrogate model, across all distances and in particular for points with inputs very far from the nearest training input. 


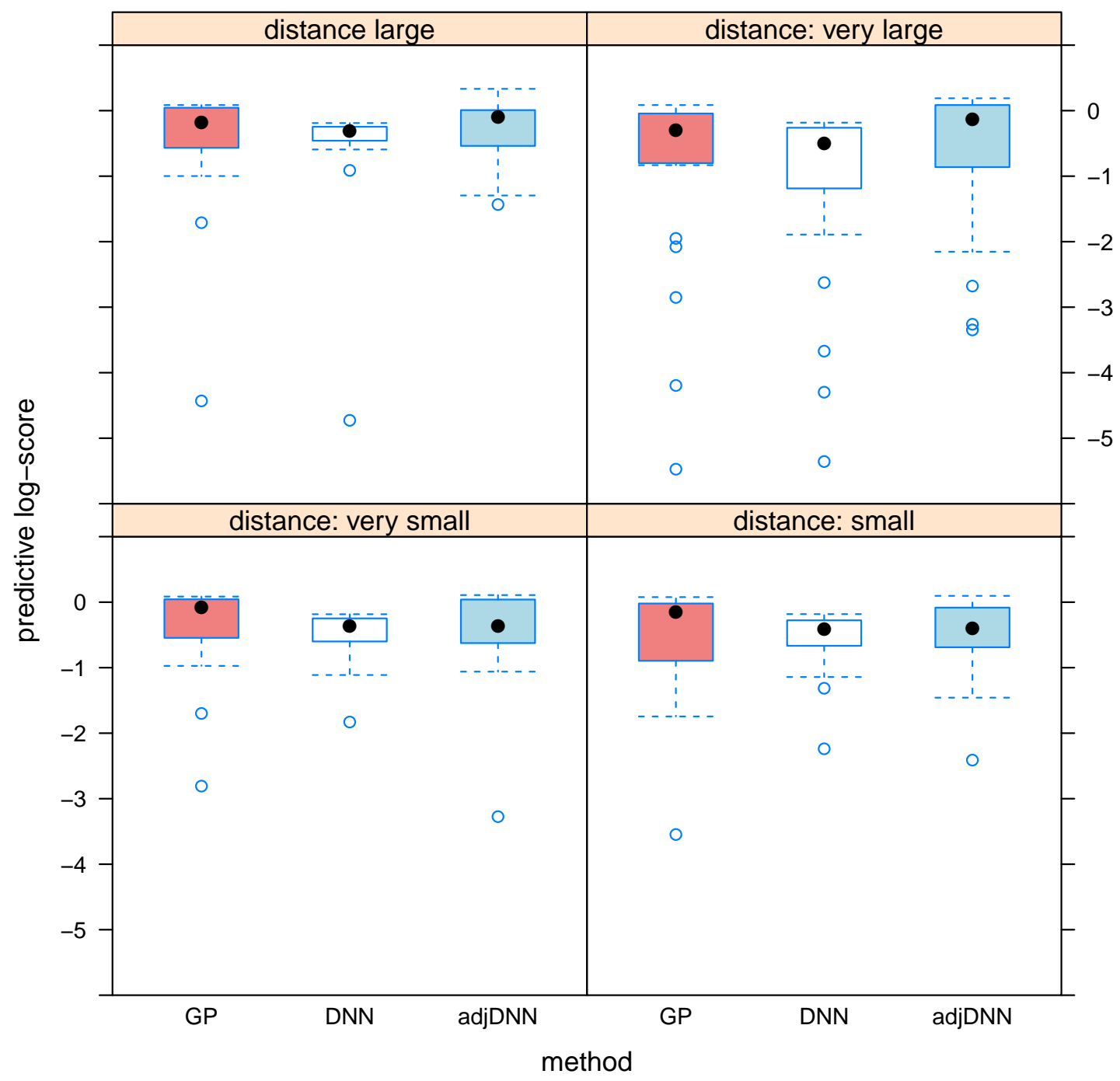

Figure 6: Boxplots of logarithmic score values across test responses by the exact GP, unadjusted DNN and DNN with adjustment for test inputs in different bins for the observed data. Different panels correspond to four distance bins based on distance from the nearest training input. The bin boundaries are equally spaced covering the range of the observed values. 


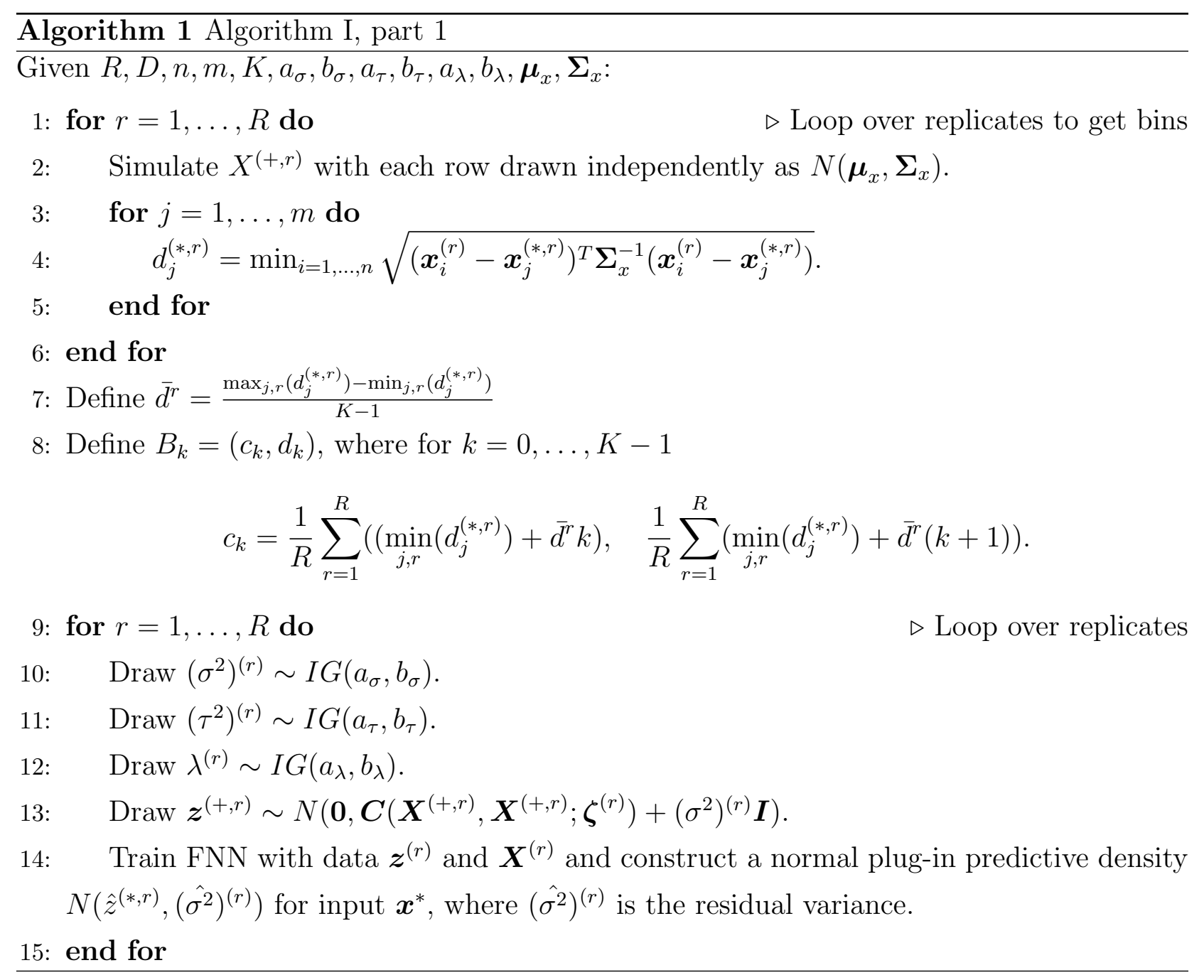


Algorithm 2 Algorithm I, part 2

16: for $k=1, \ldots, K$ do

$\triangleright$ Bin specific adjustments

17: $\quad$ Compute $\mu_{k}^{L}, \mu_{k}^{R}, \Sigma_{k}^{L}, \Sigma_{k}^{R}$ via

$$
\begin{gathered}
\mu_{k, r}^{L}=\frac{1}{m_{k}^{(r)}} \sum_{l=1}^{m} z_{l}^{(*, r)} \mathbf{1}\left(d_{l}^{(*, r)} \in B_{k}\right), \quad \mu_{k, r}^{R}=\frac{1}{m_{k}^{(r)}} \sum_{l=1}^{m} \hat{z}_{l}^{(*, r)} \mathbf{1}\left(d_{l}^{(*, r)} \in B_{k}\right), \\
\mu_{k}^{L}=\frac{1}{S} \sum_{r \in \mathcal{S}} \mu_{k, r}^{L}, \quad \mu_{k}^{R}=\frac{1}{S} \sum_{r \in \mathcal{S}} \mu_{k, r}^{R} \\
\Sigma_{k, r}^{L}=\frac{1}{m_{k}^{(r)}-1} \sum_{l=1}^{m}\left(\left(z_{l}^{(*, r)}-\mu_{k, r}^{L}\right) \mathbf{1}\left(d_{l}^{(*, r)} \in B_{k}\right)\right)^{2}, \\
\Sigma_{k, r}^{R}=\frac{1}{m_{k}^{(r)}-1} \sum_{l=1}^{m}\left(\left(\hat{z}_{l}^{(*, r)}-\mu_{k, r}^{R}\right) \mathbf{1}\left(d_{l}^{(*, r)} \in B_{k}\right)\right)^{2}+\frac{1}{m_{k}^{(r)}} \sum_{l=1}^{m}\left(\hat{\sigma}_{k}^{2}\right)^{(*, r)} \mathbf{1}\left(d_{l}^{(*, r)} \in B_{k}\right) \\
\Sigma_{k}^{L}=\frac{1}{S} \sum_{r \in \mathcal{S}} \Sigma_{k, r}^{L}, \quad \Sigma_{k}^{R}=\frac{1}{S} \sum_{r \in S} \Sigma_{k, r}^{R}
\end{gathered}
$$

where $m_{k}^{(r)}$ is the number of training samples in bin $B_{k}$ for replicate $r$.

\section{8: end for}

19: Compute $\Sigma_{k}^{R}=C_{k} C_{k}^{T}, \Sigma_{k}^{L}-\Sigma_{k, 2}^{R}=K_{k} K_{k}^{T}$, where

$$
\Sigma_{k, 2}^{R}=\frac{1}{S} \sum_{r \in \mathcal{S}} \frac{1}{m_{k}^{(r)}} \sum_{l=1}^{m}\left(\hat{\sigma}_{k}^{2}\right)^{(*, l)} \mathbf{1}\left(d_{l}^{(*, r)} \in B_{k}\right) .
$$

20:

$\triangleright$ Apply adjustments to observed data

21: Train FNN with $\boldsymbol{z}_{\text {obs }}$ and $\boldsymbol{X}_{\text {obs }}^{*}$ to obtain the plug-in predictive $N\left(\hat{z}_{\text {obs }}^{(*)}, \hat{\sigma}^{2}\right)$ at input $\boldsymbol{x}^{*}$, where $\hat{\sigma}^{2}$ is the estimated residual variance for the observed data.

22: Adjust the parameters of the plug-in Gaussian predictive

$$
\hat{z}_{\text {obs,adj }}^{(*)}=\mu_{k}^{L}-\left(\hat{z}_{\text {obs }}^{(*)}-\mu_{k}^{R}\right), \quad \hat{\sigma}_{\text {adj }}^{2}=K_{k} C_{k}^{-1} \hat{\sigma}^{2} \text { iff } d_{\text {obs }}^{*} \in B_{k},
$$

where $d_{\mathrm{obs}}^{*}=\min _{i=1, \ldots, n} \sqrt{\left(\boldsymbol{x}_{\mathrm{obs}, i}-\boldsymbol{x}^{*}\right)^{\top} \boldsymbol{\Sigma}_{x}^{-1}\left(\boldsymbol{x}_{\mathrm{obs}, i}-\boldsymbol{x}^{*}\right)}$.

\section{Discussion}

We have discussed a new approach to checking and adjustment of approximate inference algorithms. The approach is based on using the tower property of conditional expectation and law of total variance to relate prior and posterior means and covariances, and assessing any departure from equality when posterior means and covariances are approximated. Adjustments 
can be made so that the correct relationships hold after adjustment. The main strength of the approach is that it can naturally handle multivariate quantities and directly checks calibration of mean and scale parameters which are of most direct interest to users. The main weakness is that it is based on adjusting only first and second order moments of an approximation, and is unable to correct errors in more complex features relating to the shape of the posterior density.

It might be possible to apply our correction first, and then use marginal quantile-based adjustments afterwards, such as those of Rodrigues et al. (2018), to make corrections to the shape of marginal posterior densities. However, this does involve additional computation. Higher order corrections based on the law of total cumulance (Brillinger, 1969) can also be considered, but the lack of any general family of transformations of a set of particles to achieve given means, covariances and higher order moments simultaneously makes it unclear how to generalize our approach in this direction.

\section{Acknowledgements}

The authors thank Guilherme Rodrigues and Scott Sisson for sharing their code for the sum of log-normals example. David Nott was supported by a Singapore Ministry of Education Academic Research Fund Tier 1 grant (R-155-000-189-114). Nadja Klein acknowledges support through the Emmy Noether grant KL 3037/1-1 of the German research foundation (DFG).

\section{References}

Asmussen, S. and Rojas-Nandayapa, L. (2008). Asymptotics of sums of lognormal random variables with Gaussian copula. Statistics and Probability Letters, 78(16):2709-2714.

Beaumont, M. A., Zhang, W., and Balding, D. J. (2002). Approximate Bayesian computation in population genetics. Genetics, 162:2025-2035.

Blei, D. M., Kucukelbir, A., and McAuliffe, J. D. (2017). Variational inference: A review for statisticians. Journal of the American Statistical Association, 112(518):859-877.

Blum, M. G. and Tran, V. C. (2010). HIV with contact tracing: A case study in approximate Bayesian computation. Biostatistics, 11(4):644-660.

Blum, M. G. B. (2010). Approximate Bayesian computation: A non-parametric perspective. Journal of the American Statistical Association, 105:1178 - 1187. 
Blum, M. G. B. and François, O. (2010). Non-linear regression models for approximate Bayesian computation. Statistics and Computing, 20:63-75.

Blum, M. G. B., Nunes, M. A., Prangle, D., and Sisson, S. A. (2013). A comparative review of dimension reduction methods in approximate Bayesian computation. Statistical Science, 28(2):189-208.

Brillinger, D. R. (1969). The calculation of cumulants via conditioning. Annals of the Institute of Statistical Mathematics, 21(1):215-218.

Chollet, F. and Allaire, J. J. (2018). Deep Learning with R. Manning Publications Co., Greenwich, CT, USA, 1st edition.

Chung, Y., Gelman, A., Rabe-Hesketh, S., Liu, J., and Dorie, V. (2015). Weakly informative prior for point estimation of covariance matrices in hierarchical models. Journal of Educational and Behavioral Statistics, 40(2):136-157.

Cook, S. R., Gelman, A., and Rubin, D. B. (2006). Validation of software for Bayesian models using posterior quantiles. Journal of Computational and Graphical Statistics, 15(3):675-692.

Cressie, N. and Wikle, C. (2011). Statistics for Spatio-Temporal Data. Wiley.

Fenton, L. (1960). The sum of log-normal probability distributions in scatter transmission systems. IRE Transactions on Communications Systems, 8(1):57-67.

Geil, P., Million, A., Rotte, R., and Zimmerman, K. F. (1997). Economic incentives and hospitalization in Germany. Journal of Applied Econometrics, 12(3):295-311.

Gelman, A. (2017). Correction to Cook, Gelman, and Rubin (2006). Journal of Computational and Graphical Statistics, 26(4):940-940.

Gelman, A., Carlin, J., Stern, H., Dunson, D., Vehtari, A., and Rubin, D. (2013). Bayesian Data Analysis, Third Edition. Chapman \& Hall/CRC Texts in Statistical Science. Taylor \& Francis.

Jaccard, P. (1901). Étude comparative de la distribution florale dans une portion des Alpes et des Jura. Bulletin de la Société vaudoise des sciences naturelles, 37:547-579.

Lee, J. E., Nicholls, G. K., and Ryder, R. J. (2019). Calibration procedures for approximate Bayesian credible sets. Bayesian Analysis, (To Appear). 
Li, J., Nott, D. J., Fan, Y., and Sisson, S. A. (2017). Extending approximate Bayesian computation methods to high dimensions via Gaussian copula. Computational Statistics and Data Analysis, 106:77-89.

MacDonald, B., Ranjan, P., and Chipman, H. (2015). GPfit: An R package for fitting a Gaussian process model to deterministic simulator outputs. Journal of Statistical Software, 64(12):1-23.

Marin, J.-M., Pudlo, P., Robert, C. P., and Ryder, R. J. (2012). Approximate Bayesian computational methods. Statistics and Computing, 22(6):1167-1180.

Nott, D. J., Fan, Y., Marshall, L., and Sisson, S. A. (2014). Approximate Bayesian computation and Bayes linear analysis: towards high-dimensional ABC. Journal of Computational and Graphical Statistics, 23(1):65-86.

Ormerod, J. T. and Wand, M. P. (2010). Explaining variational approximations. The American Statistician, 64:140-153.

Prangle, D., Blum, M., Popovic, G., and Sisson, S. (2014). Diagnostic tools for approximate Bayesian computation using the coverage property. Australian $\&$ New Zealand Journal of Statistics, 56(4):309-329.

Rasmussen, C. E. and Williams, C. K. (2006). Gaussian processes for machine learning. The MIT Press, Cambridge, MA.

Ribeiro Jr., P. and Diggle, P. (2001). geoR: a package for geostatistical analysis. R-NEWS, $1(2): 15-18$.

Rodrigues, G., Prangle, D., and Sisson, S. (2018). Recalibration: A post-processing method for approximate Bayesian computation. Computational Statistics and Data Analysis, 126:5366.

Talts, S., Betancourt, M., Simpson, D., Vehtari, A., and Gelman, A. (2018). Validating Bayesian inference algorithms with simulation-based calibration. arXiv: 1804.06788.

Tran, M.-N., Nguyen, N., Nott, D., and Kohn, R. (2019). Bayesian deep net GLM and GLMM. Journal of Computational and Graphical Statistics, (To appear).

Wegmann, D., Leuenberger, C., and Excoffier, L. (2009). Efficient approximate Bayesian computation coupled with Markov chain Monte Carlo without likelihood. Genetics, 182(4):12071218. 
Yao, Y., Vehtari, A., Simpson, D., and Gelman, A. (2017). Yes, but did it work?: Evaluating variational inference. arXiv: 1802.02538. 